zuzustreben, die innerhalb von $s \approx 0,6 \mathrm{dem} 1$. Fall entspricht und für $s \gtrsim 0,6$ in den 3 . Fall übergeht. Soll $\omega=1$ und $\omega=a / \sqrt{s}$ bei $s=s_{\mathrm{kr}}=0,57$ stetig aneinander schließen, so muß $A=s_{\mathrm{kr}}^{2} / 8=$ 0,040 sein. Die Dichte $\varrho$ fällt an dieser Stelle von $\varrho=2$ auf $\varrho=1 \mathrm{ab}$. Es bildet sich also eine Art Kern mit dem Radius $s \approx 0,6$ und konstanter Dichte, die gleich der ursprünglichen Mittelpunktsdichte ist, aus. Die nicht zum Aufbau dieses Kerns benötigte Masse wird schließlich nach außen abfließen (vgl. Verlauf von $J$, Abb. 2).

In den hier betrachteten Zeiträumen $(\beta t \lesssim 0,1)$ kann für größere $s(s \gtrsim 1,5)$ noch keine wesentliche Veränderung der Dichte- und Geschwindigkeitsverteilung beobachtet werden. Und auch über längere Zeiten hin sind die Veränderungen der Kurven so gering, daß die Störungen von der kritischen Stelle her nicht zu vernachlässigen sind. Die Rechnungen wurden für $s \geq 1,0$ bis $\beta t=0,4$ durchgeführt, wobei Unregelmäßigkeiten an den Rändern ausgeglichen wurden.

An der kritischen Stelle selbst wird mit $\dot{v}_{\varphi}$ auch $v_{s}=-\dot{v}_{\varphi} / v_{\varphi}^{\prime}$ groß $^{-}(\mathrm{Abb} .2)$. Das hat zur Folge, daß die Annahme $v_{s} \ll v_{\varphi}, v_{s}^{\prime} \ll s \omega^{\prime}$ [vgl. Gl. II (16)] nicht mehr gültig bleibt (bei $\beta t=0,10$ ist $v_{s} \approx 6 \beta \approx 0,15$, während $s \omega^{\prime}$ ungefähr 0,25 beträgt). Es tritt zusätzliche Reibung auf, die wohl die Unstetigkeiten an der kritischen Stelle auszugleichen strebt. Wieweit sie die Bildung eines Kerns beeinflußt, ist schwer zu sagen.

\title{
Der Rotationszustand der Sonne
}

\section{Von Stefan Temesváry}

\author{
Aus dem Max-Planck-Institut für Physik, Göttingen \\ (Z. Naturforschg. 7 a, 103-120 [1952]; eingegangen am 24. September 1951)
}

Herrn Professor Werner Heisenberg zum 50. Geburtstag

\begin{abstract}
Die Gesetzmäßigkeiten der Sonnenrotation werden untersucht unter besonderer Berücksichtigung der Folgerungen, die sich aus dem ständigen Verlust an Rotationsmoment ergeben. Es wird gezeigt, daß, wenn zu dem durch die Strahlung bedingten Anteil noch eine Abbremsung durch das interplanetarische Medium hinzukommt, anschließend an die Wasserstoffkonvektionszone noch eine Zone existieren kann, in der Rotationsinstabilität herrscht. Ob diese Instabilität eine turbulente Strömungsform bedingt oder das Auftreten von stationären Zirkulationen zur Folge hat, läßt sich theoretisch nicht streng entscheiden. Die Existenz und die Gesetzmäßigkeiten der Sonnenflecken sprechen aber eher für die letztere Annahme. Die Wanderung der bevorzugten Fleckenzone innerhalb des elfjährigen Zyklus kann als die Folge einer großräumigen Zirkulation gedeutet werden, deren Geschwindigkeit zusammen mit der kinematischen Zähigkeit in der Wasserstoffkonvektionszone das an der Oberfläche beobachtete Rotationsgesetz bestimmt.

Insgesamt ergibt sich für die äußeren Teile der Sonne eine Zunahme der Winkelgeschwindigkeit nach innen hin. Im tieferen Innern dagegen hängt deren Verteilung mit ab von dem unbekannten Anfangszustand der Sonne, die noch nicht so alt ist, als daß die dort herrschende Viskosität sowie der Strahlungsstrom einen definierten Endzustand herbeigeführt haben könnten. Aus diesem Grund werden noch einige andere Kriterien herangezogen, um eine obere Grenze für die Winkelgeschwindigkeit im tieferen Innern und damit für den Gesamtdrehimpuls der Sonne zu erhalten.
\end{abstract}

$\mathrm{D}^{\circ}$ er Rotationszustand der Sonne ist bisher meist im Anschluß an allgemeinere Untersuchungen über die Sternrotation behandelt worden mit dem Ziel, die an der Oberfläche beobachtete differentielle Rotation zu erklären. Den Anlaß zu der vorliegenden Untersuchung gaben dagegen zwei Probleme der Sonnenphysik, die in letzter Zeit wieder in den Vordergrund des Interesses getreten sind, und die nicht so sehr nach dem
Rotationsgesetz an der Oberfläche fragen, sondern nach der Verteilung der Winkelgeschwindigkeit im Innern der Sonne und der sich daraus ergebenden Rotationsstabilität bzw. Instabilität bestimmter Schichten. Die beiden Problemkreise sind einmal das Sonnenflecken-Phänomen und zum andern die Kosmogonie des Sonnensystems.

Bekanntlich lassen sich die beobachteten Gesetzmäßigkeiten des Sonnenflecken-Phänomens 
weitgehend auf Grund eines von Bjerknes ${ }^{1}$ entworfenen Bildes verstehen, wonach unter der Sonnenoberfläche ein System von geschlossenen Wirbelringen innerhalb des Aktivitätszyklus umeinander kreist. Nachdem vor kurzem Bierma $n^{2}{ }^{2}$ zeigen konnte, daß auch die Entstehung von Magnetfeldern der in den Flecken beobachteten Stärke in den allgemeinen Rahmen der Bjerknesschen Vorstellungen hineinpaßt, fehlt zum vollen Verständnis des Bildes wesentlich noch eine hydrodynamische Begründung für die Entstehung dieser Zirkulationsströmungen. Nun weiß man aus Experimenten von $\mathrm{T}$ a yl or ${ }^{3}$ und anderen Autoren, daß in einem rotierenden viskosen $\mathrm{Me}$ dium bei Rotationsinstabilität stationäre meridionale Zirkulationen auftreten können, die eine gewisse Ähnlichkeit mit den von Bjerknes postulierten Wirbelringen haben. Es liegt daher nahe, zunächst einmal zu untersuchen, welche Voraussetzungen für das Auftreten einer solchen Rotationsinstabilität in den äußeren Teilen der Sonne erfüllt sein müßten. Qualitativ läßt sich dazu schon soviel sagen, daß dann die Winkelgeschwindigkeit in einem hinreichenden, noch von der thermischen Stabilität der Schichtung abhängendem Maße nach innen zunehmen müßte. Sollte die Bjerknessche Vorstellung von dem Sonnenfleckenphänomen zutreffen, so würde man daher zu der Annahme gezwungen sein, daß die Sonne im Innern um einiges rascher rotiert als an der Oberfläche.

$\mathrm{Zu}$ dem gleichen Ergebnis kommt man aber auch auf Grund kosmogonischer Überlegungen. Wenn die Sonne in einem früheren Entwicklungsstadium, etwa zur Zeit der Entstehung des Planetensystems, wie man annimmt, ein größeres Rotationsmoment besessen hat als heute (wofür u. a. auch die Beobachtung größerer Rotationsgeschwindigkeiten an frühen Sterntypen spricht), so ist die Frage, auf welche Weise sie dieses Rotationsmoment inzwischen verloren hat. - Nun. ist es $\mathrm{Lüst}{ }^{4}$ gelungen, in Fortführung der Untersuchungen v. Weizs ä ckers einen Mechanismus

1 V. Bjerknes, Astrophysic. J. 64, 93 [1926]; vgl. dazu W. Grotrian Z. angew. Physik 2, 376 [1950].

${ }_{2}$ L. B i erm a n n, Z. Naturforschg. 5 a, 65 [1950].

3 G.I.T a y l o r, Philos. Trans. Roy. Soc. [London], Ser. A 223, 289 [1923].

4 R. Lüst, Z. Naturforschg. 7 a [1952] (in Vorbereitung).

5 M. Schwarzschild Astrophysic. J.106, 427 [1947].

6 In früheren Arbeiten $6 \mathrm{a}, 6 \mathrm{~b}$ wurde sonst diesen Kräften durch die Annahme Rechnung getragen, da $B$ anzugeben, durch den die Sonne nicht nur in ,vorgeschichtlicher" Zeit abgebremst worden ist, sondern wohl auch heute noch laufend Drehimpuls an das sie umgebende interplanetarische Medium abgibt und zwar in einem Betrag, der etwa von der gleichen Größenordnung ist wie der ständige Verlust an Rotationsmoment, den sie durch ihre Ausstrahlung erleidet. Da diese Abbremsung sich vorzugsweise auf die äußeren Teile erstrecken wird, kann man auch aus diesem Grunde eine Zunahme der Winkelgeschwindigkeit nach innen erwarten. Wenn sich bei einer numerischen Abschätzung der Einfluß dieser Abbremsung der äußeren Teile der Sonne als hinreichend groß erweist, könnte es sein, daß hiermit die noch fehlende hydrodynamische Begründung des Sonnenfleckenphänomens gefunden wäre.

Diese Vermutung, daß das Sonneninnere rascher rotiere als die Oberfläche, steht aber zunächst im Widerspruch zu einem Resultat von Schwarzschild ${ }^{5}$, der auf Grund bestimmter vereinfachender Annahmen auf numerischem Wege ein Modell der rotierenden Sonne berechnen konnte und eine Abnahme der Winkelgeschwindigkeit nach innen hin fand. Es läßt sich jedoch zeigen, daß einige der von. Schwarzschild gemachten Voraussetzungen nicht auf alle Teile der Sonne angewandt werden können bzw. unserer wesentlich anders gearteten Fragestellung nicht entsprechen. Insbesondere wird von ihm, wie bei ähnlichen Untersuchungen teilweise auch von anderen Autoren, folgendes angenommen: 1. Meridionale Strömungen können sowohl hinsichtlich des Transportes von Rotationsmoment wie auch für den Energietransport vernachlässigt werden. 2. In dem im Strahlungsgleichgewicht befindlichen Hauptteil der Sonne kann die Viskosität vernachlässigt werden. Ebenso werden praktisch die viskosen Kräfte in der Wasserstoffkonvektionszone vernachlässigt ${ }^{6}$. 3. Die Verteilung der Winkelgeschwindigkeit ist im ganzen Stern stationär, d. h. unabhängig von der Zeit gegeben.

Es leuchtet sofort ein, daß ein Ansatz, der nach

die Komponente des Gradienten der Winkelgeschwindigkeit in Richtung der Oberflächennormalen verschwindet. Diese Randbedingung hat $\mathrm{S} \mathrm{chw}$ a r z schild im vorliegenden Fall nicht mehr frei, da er seine Integration im Innern an den konvektiven Kern der Sonne in einer definierten Weise anschließt. 6a M. Schwarzschild, Astrophysic. J. 95, 441 [1942].

6b G. R anders, Astrophysic. J. 94, 109 [1941]. 
der ersten Annohme meridionale Zirkulationen ausschließt, niemals zu einer hydrodynamischen Begründung der Bjerknesschen. Vorstellung vom Sonnenfleckenphänomen führen kann. Die Tatsache, daß die von der Theorie gemäß der Verallgemeinerung des $\mathbf{v}$. Zeipelschen Theorems geforderten meridionalen Strömungen in der Sonne außerordentlich langsam sind 7,8 , schließt nicht aus, daß es noch eine andere Ursache gibt, die schnellere Zirkulationen zur Folge haben kann.

Hierzu wie auch bezüglich der zweiten Annahme müssen einige ältere Abschätzungen und die sich daraus ergebenden Konsequenzen in Erinnerung gebracht werden. Aus den Untersuchungen von Jea ns ${ }^{9,10}$ weiß man, daß außer der Viskosität und den meridionalen Strömungen auch der Strahlungsstrom Einfluß auf die Verteilung der Winkelgeschwindigkeit haben kann. Dieser stellt nach dem Äquivalenzprinzip zugleich èinen Massenstrom dar, der ebenso wie eine materielle Strömung Drehimpuls transportiert und auf einen Ausgleich des Rotationsmomentes hinwirkt. Außerdem ist seit längerem bekannt, daß alle drei Größen im tieferen Innern der Sonne zwar die ursprüngliche Verteilung nicht wesentlich geändert haben können, in den äußeren Teilen dagegen weder die Viskosität noch die Bremswirkung der Strahlung ohne weiteres vernachlässigt werden dürfen, und zwar überwiegt hier der Einfluß des Strahlungsstromes noch weitaus den der Strahlungsviskositäti1. Dies wird einen Zustand ausgeglichenen Rotationsmomentes zur Folge haben, so daß in den äußeren Teilen der Sonne, soweit sie sich im Strahlungsgleichgewicht befinden, die Winkelgeschwindigkeit nach innen hin zunehmen muß. In der Wasserstoffkonvektionszone (WKZ) kann zwar eine völlig isotrope turbulente Viskosität praktisch starre Rotation bewirken, tritt aber noch eine äußere Abbremsung durch Reibung hinzu, so muß auch in diesem Fall ein zwar kleiner, aber negativer Gradient der Winkelgeschwindigkeit herrschen.

Die dritte der genannten Annahmen bedarf bezüglich der aus ihr zu ziehenden Schlüsse einer gewissen Einschränkung. Soweit wenigstens eine der die Verteilung der Winkelgeschwindigkeit be-

7 A. S. Eddington, Monthly Notices Roy. astronom. Soc. 90, 54 [1929].

8 E. J. Ö pik, Proc. Roy. Irish Acad., Sect. A 53, 1 [1949]; vgl. auch P. A. Sweet, Monthly Notices Roy. astronom. Soc. 110, 548 [1950]. stimmenden Größen groß genug ist, um innerhalb eines Zeitraumes, in dem sich diese Größe selbst nicht wesentlich ändert, eine endgültige und damit stationäre Verteilung herbeigeführt zu haben, wird sich das Rotationsgesetz auf Grund der gemachten Annahme und bei genauer Kenntnis der bestimmenden Größen sowie der notwendigen Randbedingung des Problems angeben lassen. Wenn aber z. B. im tieferen Innern der Sonne die bestimmenden Größen sämtliche zu klein sind, um die Verteilung der Winkelgeschwindigkeit zu beeinflussen, wird diese zwar ebenfalls stationär sein; dies besagt aber nur, daß dort praktisch noch der Anfangszustand herrschen wird, der deswegen keineswegs identisch zu sein braucht mit der aus einer Integration der Bewegungsgleichung erhaltenen stationären Lösung, die bei verschwindend kleinen Koeffizienten einem erst nach beliebig langer Zeit erreichbarem Endzustand entspricht. In diesem Fall wird die Frage nach der gegenwärtigen Verteilung der Winkelgeschwindigkeit schon zu einem kosmogonischen Problem.

Und da sich damit auch der Gesamtdrehimpuls der Sonne nicht eindeutig wird angeben lassen, bleibt nur die Möglichkeit, ihn einzuschränken zwischen einem Maximalwert, der noch mit gewissen Beobachtungstatsachen (Perihelverschiebung der Merkurbahn, Kugelgestalt der Sonnenoberfläche) verträglich ist und einem Mindestwert, der - bei Ausschließung des kosmogonisch unwahrscheinlichen Falles einer nach innen abnehmenden Winkelgeschwindigkeit - einer starren Rotation des Sonnenkernes mit der an der inneren Grenze des definierten Bereiches erreichten Geschwindigkeit entspricht.

Unter diesen Umständen wird man von vornherein keine allgemeine und vollständige Lösung des Problems erwarten dürfen. Es kann vielmehr nur versucht werden, ausgehend von einem. möglichst allgemeinen Ansatz die geschilderten $\mathrm{Zu}$ sammenhänge zu klären, auch wenn dabei das Endergebnis mehr den Charakter einer $\mathrm{Ab}$ schätzung haben wird. Der Gang der Untersuchung läßt sich in fünf Schritte unterteilen: Im ersten Abschnitt werden die Bewegungsgleichungen und ein Ausdruck für den Drehimpuls-

9 J. H. J eans, Monthly Notices Roy. astronom. Soc. 86, 328, 444 [1926].

10 J. H. Jeans, Astronomy and Cosmogony, University Press, Cambridge 1928.

${ }_{11}$ H. V ogt, Aufbau und Entwicklung der Sterne, $\Lambda$ kadem. Verlagsgesellschaft, Leipzig 1943 Kap. XIII. 
strom abgeleitet. - Im zweiten Abschnitt werden die Zeitskala, innerhalb deren sich eine stationäre Verteilung der Winkelgeschwindigkeit einstellt, und die Oberflächenbedingungen erörtert. Dabei sind die letzteren wesentlich durch den Verlust an Rotationsmoment gegeben, den die Sonne pro Zeiteinheit erleidet. - Im dritten Abschnitt wird damit sodann auf Grund der numerischen Daten des Modelles einer nichtrotierenden Sonne das Rotationsgesetz der äußeren Teile genähert abgeleitet und die Stabilität dieser Schichten untersucht. - Im vierten Abschnitt wird das dabei erhaltene Ergebnis mit den an der Oberfläche beobachteten Erscheinungen in Zusammenhang gebracht und eine hydrodynamische Deutung des Sonnenfleckenphänomens versucht. - Im fünften Abschnitt schließlich werden in der erwähnten Weise die Grenzen bestimmt, innerhalb deren das Rotationsgesetz der inneren Teile der Sonne und damit ihr Gesamtdrehimpuls liegen muß.

\section{Die Bewegungsgleichungen}

Die Navier-Stokessche Gleichung für die Bewegung eines viskosen kompressiblen Mediums

12 Es war bisher meist üblich, den Viskositätskoeffizienten als im ganzen Stern konstant anzunehmen 6b, 12a. Die numerische Rechnung wird aber zeigen, daß die Glieder mit grad $\eta$ keineswegs vernachlässigt werden dürfen, sondern vor allem in den äußersten Teilen der Sonne eine ganz wesentliche Rolle spielen können.

12a G. R and ers, Astrophysic. J. 95, 454 [1942]. in einem Schwerefeld lautet bei Berücksichtigung einer Ortsabhängigkeit der Viskosität ${ }^{12}$

$-\varrho\left(\frac{\partial \mathfrak{v}}{\partial t}+\frac{1}{2} \operatorname{grad} \mathfrak{v}^{2}-[\mathfrak{v} \operatorname{rot} \mathfrak{v}]+\operatorname{grad} V\right)=$ $\operatorname{grad} p-\frac{4}{3} \eta \operatorname{grad} \operatorname{div} \mathfrak{v}+\eta \operatorname{rot} \operatorname{rot} \mathfrak{v}$

$-\frac{1}{3} \operatorname{div} \mathfrak{v} \operatorname{grad} \eta-\operatorname{rot}[\mathfrak{v} \operatorname{grad} \eta]$

$-\operatorname{grad}(\mathfrak{v} \operatorname{grad} \eta)+\mathfrak{v} \Delta \eta$.

Dabei ist $p$ der Druck, $\varrho$ die Dichte, $\mathfrak{v}$ die Geschwindigkeit, $\boldsymbol{V}$ das Gravitationspotential und $\eta$ der Viskositätskoeffizient $\left[\mathrm{g} \mathrm{cm}^{-1} \mathrm{sec}^{-1}\right]$.

Für den Koeffizienten der Volumenviskosität wurde

angenommen.

$$
\lambda=-\frac{2}{3} \eta
$$

In Kugelkoordinaten $(r, \vartheta, \varphi)$ erhält man aus (1. 1) unter der Annahme von Rotationssymmetrie mit

$$
\begin{gathered}
\frac{\partial}{\partial_{\varphi}}=0, \\
v_{\varphi}=\omega r \sin \vartheta, \\
\operatorname{div} \mathfrak{v}=\frac{\partial v_{r}}{\partial r}+\frac{2}{r} v_{r}+\frac{1}{r} \frac{\partial v_{\vartheta}}{\partial \vartheta}+\frac{1}{r} v_{\vartheta} \operatorname{ctg} \vartheta, \\
\Delta \eta=\frac{\partial^{2} \eta}{\partial r^{2}}+\frac{2}{r} \frac{\partial \eta}{\partial r}+\frac{1}{r^{2}} \frac{\partial^{2} \eta}{\partial \vartheta^{2}}+\frac{1}{r^{2}} \frac{\partial \eta}{\partial \vartheta} \operatorname{ctg} \vartheta
\end{gathered}
$$

drei Bewegungsgleichungen und zwar für die $r$-Komponente

$$
\begin{aligned}
\varrho \frac{\partial v_{r}}{\partial t}= & \varrho \omega^{2} r \sin ^{2} \vartheta-\varrho \frac{\partial V}{\partial r}-\frac{\partial p}{\partial r}-\varrho\left(v_{r} \frac{\partial v_{r}}{\partial r}+v_{\vartheta} \frac{1}{r} \frac{\partial v_{r}}{\partial \vartheta}-\frac{1}{r} v_{\vartheta}^{2}\right) \\
& +\frac{4}{3} \eta\left(\frac{\partial^{2} v_{r}}{\partial r^{2}}+\frac{2}{r} \frac{\partial v_{r}}{\partial r}-\frac{2}{r^{2}} v_{r}\right)+\eta\left(\frac{1}{r^{2}} \frac{\partial^{2} v_{r}}{\partial \vartheta^{2}}+\frac{1}{r^{2}} \frac{\partial v_{r}}{\partial \vartheta} \operatorname{ctg} \vartheta\right) \\
& +\frac{1}{3} \eta\left(\frac{1}{r} \frac{\partial^{2} v_{\vartheta}}{\partial r \partial \vartheta}+\frac{1}{r} \frac{\partial v_{\vartheta}}{\partial r} \operatorname{ctg} \vartheta\right)-\frac{7}{3} \eta\left(\frac{1}{r^{2}} \frac{\partial v_{\vartheta}}{\partial \vartheta}+\frac{1}{r^{2}} v_{\vartheta} \operatorname{ctg} \vartheta\right) \\
& +\frac{4}{3} \frac{\partial \eta}{\partial r}\left(\frac{\partial v_{r}}{\partial r}-\frac{1}{r} v_{r}\right)-\frac{2}{3} \frac{\partial \eta}{\partial r}\left(\frac{1}{r} \frac{\partial v_{\vartheta}}{\partial \vartheta}+\frac{1}{r} v_{\vartheta} \operatorname{ctg} \vartheta\right) \\
& +\frac{\partial \eta}{\partial \vartheta}\left(\frac{1}{r} \frac{\partial v_{r}}{\partial r}+\frac{1}{r^{2}} \frac{\partial v_{r}}{\partial \vartheta}-\frac{1}{r^{2}} v_{\vartheta}\right)
\end{aligned}
$$

die $\vartheta$-Komponente

$$
\begin{aligned}
\varrho \frac{\partial v_{\vartheta}}{\partial t}= & \varrho \omega^{2} r \sin \vartheta \cos \vartheta-\varrho \frac{1}{r} \frac{\partial V}{\partial \vartheta}-\frac{1}{r} \frac{\partial p}{\partial \vartheta}-\varrho\left(v_{r} \frac{\partial v_{\vartheta}}{\partial r}+v_{\vartheta} \frac{1}{r} \frac{\partial v_{\vartheta}}{\partial \vartheta}+\frac{1}{r} v_{r} v_{\vartheta}\right) \\
& +\frac{4}{3} \eta\left(\frac{1}{r^{2}} \frac{\partial^{2} v_{\vartheta}}{\partial \vartheta^{2}}+\frac{1}{r^{2}} \frac{\partial v_{\vartheta}}{\partial \vartheta} \operatorname{ctg} \vartheta-\frac{1}{r^{2}} \frac{v_{\vartheta}}{\sin ^{2} \vartheta}\right)+\eta\left(\frac{\partial^{2} v_{\vartheta}}{\partial r^{2}}+\frac{2}{r} \frac{\partial v_{\vartheta}}{\partial r}\right) \\
& +\frac{4}{3} \frac{\partial \eta}{\partial \vartheta}\left(\frac{1}{r^{2}} \frac{\partial v_{\vartheta}}{\partial \vartheta}-\frac{1}{r^{2}} \frac{v_{\vartheta}}{2} \operatorname{ctg} \vartheta\right)-\frac{2}{3} \frac{\partial \eta}{\partial \vartheta}\left(\frac{1}{r} \frac{\partial v_{r}}{\partial r}-\frac{1}{r^{2}} v_{r}\right) \\
& +\frac{\partial \eta}{\partial r}\left(\frac{1}{r} \frac{\partial v_{r}}{\partial \vartheta}+\frac{\partial v_{\vartheta}}{\partial r}-\frac{1}{r} v_{\vartheta}\right),
\end{aligned}
$$


die $\varphi$-Komponente

$$
\begin{aligned}
\varrho r \frac{\partial \omega}{\partial t}= & -\varrho\left\{v_{r}\left(r \frac{\partial \omega}{\partial r}+2 \omega\right)+v_{\vartheta}\left(\frac{\partial \omega}{\partial \vartheta}+2 \omega \operatorname{ctg} \vartheta\right)\right\} \\
& +\eta\left\{r \frac{\partial^{2} \omega}{\partial r^{2}}+4 \frac{\partial \omega}{\partial r}+\frac{1}{r} \frac{\partial^{2} \omega}{\partial \vartheta^{2}}+3 \frac{1}{r} \frac{\partial \omega}{\partial \vartheta} \operatorname{ctg} \vartheta\right\} \\
& +\frac{\partial \eta}{\partial r} r \frac{\partial \omega}{\partial r}+\frac{\partial \eta}{\partial \vartheta} \frac{1}{r} \frac{\partial \omega}{\partial \vartheta}
\end{aligned}
$$

Davon werden wir im wesentlichen die letzte Gleichung (1.4) benötigen und wollen zunächst mit ihrer Hilfe einen Ausdruck für den Strom von Drehimpuls in bezug auf die $\varphi$-Achse herleiten, der pro Zeit- und Flächeneinheit durch die Oberfläche eines Volumenelementes fließt. Bezeichnen wir ihn als einen Vektor $\Im$ definiert durch $-\frac{\partial}{\partial t} \int \varrho \omega r^{2} \sin ^{2} \vartheta \mathrm{d} \tau=\oint \Im \mathrm{d} f=\int \operatorname{div} \Im \mathrm{d} \tau,(1.5)$ so gilt

$$
\operatorname{div} \mathfrak{\Im}=-\varrho r^{2} \sin ^{2} \vartheta \frac{\partial \omega}{\partial t}-\omega r^{2} \sin ^{2} \vartheta \frac{\partial \varrho}{\partial t},
$$

und wir erhalten, indem wir $\partial \omega / \partial t$ aus (1.4) und $\partial \varrho / \partial t$ aus der Kontinuitätsbedingung

$$
-\frac{\partial}{\partial t} \int \varrho \mathrm{d} \tau=\oint \varrho \mathfrak{v} \mathrm{d} f=\int \operatorname{div} \varrho \mathfrak{v} \mathrm{d} \tau,
$$

d. h.

$$
-\frac{\partial \varrho}{\partial t}=\varrho \operatorname{div} \mathfrak{v}+(\mathfrak{v} \operatorname{grad} \varrho),
$$

in (1.6) einsetzen und berücksichtigen, daß bei Rotationssymmetrie

$\operatorname{div} \Im=\frac{\partial}{\partial r} I_{r}+\frac{2}{r} I_{r}+\frac{1}{r} \frac{\partial}{\partial \vartheta} I_{\vartheta}+\frac{1}{r} I_{\vartheta} \operatorname{ctg} \vartheta(1.9)$

ist, für die beiden Komponenten des Drehimpuls- stromes in Utbereinstimmung mit $\mathrm{Jeans}^{10}$ (l. c., p. 275)

$$
I_{r}=\varrho \omega r^{2} \sin ^{2} \vartheta v_{r}-\eta r^{2} \sin ^{2} \vartheta \frac{\partial \omega}{\partial r}
$$

und

$$
I_{\vartheta}=\varrho \omega r^{2} \sin ^{2} \vartheta v_{\vartheta}-\eta r \sin ^{2} \vartheta \frac{\partial \omega}{\partial \vartheta}
$$

\section{Zeitskala und Oberflächen- bedingungen}

Will man die räumliche Verteilung der Winkelgeschwindigkeit $\omega$ aus Gl. (1.4) erschließen, so ist zu beachten, daß diese vom Typus der Wärmeleitungsgleichung ist. D. h., die derzeitige räumliche Verteilung der Winkelgeschwindigkeit hängt noch mit ab von dem unbekannten Anfangszustand und läßt sich daher nur angeben in einem Bereich, in dem entweder die kinematische Zähigkeit $v=\eta / \varrho$ oder die meridionale Strömungsgeschwindigkeit $v_{m}\left(v_{r}, v_{\vartheta}\right)$ oder beide zusammen gro $\beta$ genug sind, um in einem Zeitraum, der klein ist gegenüber dem Entwicklungsalter der Sonne $\left(\approx 3 \cdot 10^{9} \mathrm{Jahre}=10^{17} \mathrm{sec}\right)$, einen definierten Endzustand herbeigeführt zu haben. In diesem Falle kann $\operatorname{man} \partial \omega / \partial t=0$ setzen und erhält für (1.4) nach einiger Umformung

$$
\begin{aligned}
\frac{\partial \ln \omega}{\partial t}=\frac{v}{r^{2}} & \left\{\frac{\partial}{\partial \ln r}\left(\frac{\partial \ln \omega}{\partial \ln r}\right)+\left(\frac{\partial \ln \omega}{\partial \ln r}\right)^{2}+\left(3+\frac{\partial \ln \eta}{\partial \ln r}\right) \frac{\partial \ln \omega}{\partial \ln r}\right. \\
& \left.+\frac{\partial}{\partial \vartheta}\left(\frac{\partial \ln \omega}{\partial \vartheta}\right)+\left(\frac{\partial \ln \omega}{\partial \vartheta}\right)^{2}+\left(3 \operatorname{ctg} \vartheta+\frac{\partial \ln \eta}{\partial \vartheta}\right) \frac{\partial \ln \omega}{\partial \vartheta}\right\} \\
& -\frac{v_{m}}{r}\left\{\frac{v_{r}}{v_{m}}\left(2+\frac{\partial \ln \omega}{\partial \ln r}\right)+\frac{v_{\vartheta}}{v_{m}}\left(2 \operatorname{ctg} \vartheta+\frac{\partial \ln \omega}{\partial \vartheta}\right)\right\}=0
\end{aligned}
$$

unter der Voraussetzung, daß

$$
\frac{r^{2}}{v} \ll 10^{17} \mathrm{sec}
$$

bzw. $\quad \frac{r}{v_{m}} \ll 10^{17} \mathrm{sec}$.
Rein örtlich kann dabei der momentane Einfluß einer meridionalen Strömung noch um so größer und die damit verknüpfte Zeitskala entsprechend um so kleiner sein, je mehr die Verteilung der Winkelgeschwindigkeit vom Falle eines ausge- 
glichenen Rotationsmomentes abweicht, während der Einfluß der Viskosität und die Länge der entsprechenden Zeitskala davon abhängt, wie weit der örtliche Gradient von $\omega$ vom Falle der starren Rotation abweicht und wie groß der örtliche Gradient des Viskositätskoeffizienten selbst ist.

Die partielle Differentialgleichung (2.1) ist vom elliptischen Typ. Die Größen $\eta, \varrho, v_{r}$ und $v_{\vartheta}$ als Funktionen von $r$ und $\vartheta$ als bekannt vorausgesetzt, genügt es dann, um $\omega$ zu ermitteln, wenn als Randbedingung der Gradient von $\omega$ in Richtung dèr Oberflächennormalen gegeben ist. Physikalisch wird $[\partial \omega / \partial r]_{0}$ (der Index Null bezeichnet im folgenden stets den Wert an der Oberfläche) durch den Zustand der oberflächennahen Schichten sowie durch den Fluß an Rotationsmoment durch die Oberfläche bestimmt.

Dieser Drehimpulsstrom durch die Oberfläche besteht zunächst aus einem konvektiven Anteil, der durch den Nettostrom an Strahlungsenergie $H_{r}$ pro Zeit- und Flächeneinheit gegeben ist. In den äußeren energiequellenfreien Teilen der Sonne beträgt er nach (1.10)

$\varrho \omega r^{2} \sin ^{2} \vartheta v_{r}=\frac{H_{r}}{c^{2}} \omega r^{2} \sin ^{2} \vartheta=\frac{\omega L}{4 \pi c^{2}} \sin ^{2} \vartheta$,

wobei $L=10^{33,60} \mathrm{erg} \mathrm{sec}^{-1}$ die Gesamtleuchtkraft der Sonne ist ${ }^{13}$. Durch die Integration über die ganze Oberfläche erhält man damit den Verlust an Rotationsmoment, den die Sonne pro Zeiteinheit infolge ihrer Ausstrahlung erleidet:

$$
\frac{2}{3} \frac{L}{c^{2}} \omega_{0} R^{2}=10^{28,6} \mathrm{~cm}^{2} \mathrm{~g} \mathrm{sec}^{-2}
$$

mit $\omega_{0}=10^{-5,54} \mathrm{sec}^{-1}$, der Winkèlgeschwindigkeit am Äquator, und $R=10^{10,84} \mathrm{~cm}$, dem Sonnenradius. Die entsprechende Zeitskala ist gegeben durch

$$
\frac{4 \pi R^{3} c^{2}}{L} \varrho_{0} \approx 10^{14} \mathrm{sec}
$$

mit $\varrho_{0} \approx 10^{-7} \mathrm{~g} \mathrm{~cm}^{-3}$. Demnach ist in der Nähe der Oberfläche die Bedingung (2.2) mit Sicherheit erfüllt.

Hier hinzu kommt nun noch ein weiterer Anteil des Drehimpulsstromes, der auf der Reibung zwischen der Sonne und dem sie umgebenden interplanetarischem Gas beruht. Da dieses die

13 Der Verlust durch Korpuskular- und Höhenstrahlung wurde gegenüber der Ausstrahlung von sichtbarem Licht vernachlässigt.
Eigenschaften eines Plasmas besitzt, kann man annehmen, daß es durch das Magnetfeld der Sonne nahezu starr mitgeführt wird (wofür auch die Beobachtungen an der Korona sprechen!), und zwar bis zu einer bestimmten Entfernung, in der dann durch turbulente Reibung Drehimpuls an das weiter außen befindliche Medium abgegeben wird. Eine quantitative Abschätzung hierzu hat L üst ${ }^{4}$ gegeben, nämlich wie folgt:

Es wird angenommen, die Sonne besitze ein Dipolmoment $d=10^{33} \mathrm{Gau} ß \mathrm{~cm}^{3}$, was einer Polfeldstärke von etwa einem Gauß entspricht. Der Abfall des Feldes erfolge mit $H \sim r^{-3}$, dann ist die magnetische Energiedichte

$$
\frac{H^{2}}{8 \pi} \approx \frac{d^{2}}{8 \pi r^{6}} .
$$

Die praktisch starre Mitführung des Plasmas reiche bis zu einer Entfernung, in der die magnetische Energiedichte soweit abgenommen hat, daß sie mit der turbulenten Energiedichte

$$
\frac{1}{2} \varrho w^{2} \approx \frac{1}{2} \varrho f^{2} \omega^{2} r^{2} \sin ^{2} \vartheta
$$

vergleichbar wird. Dabei ist $f$ ein Zahlenfaktor, der das Verhältnis der Turbulenzgeschwindigkeit zur Rotationsgeschwindigkeit angibt. Wir rechnen mit $f=1 / 10$. Die beiden Ausdrücke einander gleichgesetzt gibt mit $\omega=\omega_{0}=10^{-5,54} \mathrm{sec}^{-1}$, der Winkelgeschwindigkeit am Sonnenäquator, und $\varrho \approx 10^{-22} \mathrm{~g} \mathrm{~cm}^{-3}$, der Dichte im interplanetarischen Raum,

$$
r \approx 10^{12,5}(\sin \vartheta)^{-1 / 4}[\mathrm{~cm}] .
$$

Das ist eine Entfernung, die für $\vartheta=90^{\circ}$ etwas innerhalb der Merkurbahn $\left(r=10^{12,76} \mathrm{~cm}\right)$ liegt.

Macht man weiter für den turbulenten Aus. tauschkoeffizienten den Ansatz

$$
\eta_{T} \approx \varrho w l \approx \varrho f \omega r \sin \vartheta \cdot f r \sin \vartheta
$$

und ersetzt in (1.10) den Differentialquotienten $\partial \omega / \partial r$ durch das Verhältnis $\omega / r$, so ist der Reibungsanteil des Drehimpulsstromes pro Zeit- und Flächeneinheit in der durch (2.8) gegebenen Entfernung

$$
-\eta r^{2} \sin ^{2} \vartheta \frac{\partial \omega}{\partial r} \approx \varrho f^{2} \omega^{2} r^{3} \sin ^{4} \vartheta .
$$

Und da, starre Mitführung des dazwischen liegenden Mediums vorausgesetzt, der entsprechende Wert an der Sonnenoberfläche um den Faktor 
$(r / R)^{2}$. größer sein muß, ist unter den gemachten Voraussetzungen die gesuchte Randbedingung gegeben durch

$$
\left[\frac{\partial \ln \omega}{\partial \ln r}\right]_{0} \approx-10^{0,44} \frac{(\sin \vartheta)^{3 / 4}}{\eta_{0}} .
$$

Der Exponent von $\sin \vartheta$ ist bei der Unsicherheit des Ansatzes natürlich nicht verbürgt.

Durch Integration über die ganze Sonnenoberfläche erhält man dementsprechend einen gesamten Drehimpulsverlust pro Zeiteinheit infolge äußerer Reibung von etwa $10^{28,3} \mathrm{~cm}^{2} \mathrm{~g} \mathrm{sec}^{-2}$ also die gleiche Größenordnung wie nach (2.4) infolge der Ausstrahlung.

Bei Annahme einer höheren interplanetarischen Dichte (maximal 10-21 $\mathrm{g} \mathrm{cm}^{-3}$, vgl. Lü s t ${ }^{4}$ ) erhöht sich dieser Wert proportional $\varrho^{3 / 8}$. Allerdings könnte dann der Fall eintreten, daß nach (2.8) $r \sim \varrho^{-1 / 8}$ kleiner wird als der Radius der der angenommenen Winkelgeschwindigkeit entsprechenden Kepler-Bahn $\left(r=10^{12,40} \mathrm{~cm}\right)$, so daß keine Abbremsung der Sonne mehr stattfindet, sondern umgekehrt eine des sie umgebenden Mediums, das demzufolge in die Sonne abströmen müßte. Das Funktionieren des angegebenen Mechanismus hängt demnach davon ab, daß das Magnetfeld stark genug ist im Verhältnis zur interplanetarischen Dichte. D. h., es muß ungefähr gelten

$$
d>10^{43} \sqrt{4 \pi \varrho_{\text {interpl. }}}\left[\mathrm{Gau} \beta \mathrm{cm}^{3}\right] .
$$

\section{Das Rotationsgesetz der äußeren Teile der Sonne Die Instabile Zone}

Zunächst werde gezeigt, daß eine äußere Abbremsung der Sonne durch das interplanetarische Medium die Existenz einer rotationsinstabilen Zone unterhalb der thermisch instabilen Schicht der Wasserstoffkonvektionszone (WKZ) bewirken kann.

Bei der infolge des turbulenten Austausches hohen Viskosität der WKZ genügt nach (2.11) schon ein sehr kleiner negativer Gradient der Winkelgeschwindigkeit, um den geforderten Transport von Drehimpuls bis an die Oberfläche zu besorgen: Der gesamte Betrag an Drehimpuls, der pro Zeiteinheit an der Oberfläche abgenommen wird, ist nach der gemachten Abschätzung aber so groß, daß er nicht allein der WKZ entstammen wird, die nur einen kleinen Bruchteil der Sonnenmasse umfaßt, sondern aus einer Tiefe kommen muß, die wenigstens so groß ist, daß der Gesamtdrehimpuls der außerhalb befindlichen Teile von der Größenordnung $10^{28,3} \cdot 10^{17}=10^{45,3} \mathrm{~cm}^{2} \mathrm{~g} \mathrm{sec}^{-1}$ ist. Für dieses Gebiet muß daher nach (2.11) gelten

$$
\frac{\partial \ln \omega}{\partial \ln r}=-10^{0,44} \frac{\omega_{0}}{\omega}\left(\frac{R}{r}\right)^{3} \frac{(\sin \vartheta)^{3 / 4}}{\eta} .
$$

D. h., der durch die innere Reibung bewirkte Anteil des pro Zeiteinheit durch eine Niveaufläche nach außen fließenden Drehimpulsstromes sei in diesen äußeren Teilen praktisch konstant. Dann hängt der Betrag des dadurch geforderten negativen Gradienten von $\omega$ wesentlich von der Viskosität ab. Wenn die : Schichten unterhalb der WKZ thermisch stabil sind, hat man dort zunächst nur mit der molekularen Viskosität und der Strahlungsviskosität zu rechnen. Da diese sehr viel kleiner sind als die turbulente Viskosität der WKZ, kann aber der Fall eintreten, daß der dann geforderte Gradient von $\omega$ so groß wird, daß Rotationsinstabilität eintritt. Um dies zu untersuchen, benötigt man zweierlei, nämlich einmal ein geeignetes Stabilitätskriterium und dann ein Modell für die Temperatur- und Dichteverteilung im Sonneninnern, um den Viskositätskoeffizienten zu berechnen.

Ein exaktes allgemeines Kriterium für Stabilität gegen jede mögliche Art von Störungen ist noch nicht gegeben worden. Es möge daher genügen, wenn man umgekehrt die Bedingungen für das Auftreten einiger naheliegender Formen von Instabilität betrachtet, indem man z. B. die Energiebilanz für die radiale bzw. tangentiale Verrückung eines bestimmten Volumenelementes aufstellt. Dabei ist $\mathrm{zu}$ berücksichtigen, daß eine $u$. U. herrschende thermisch stabile Schichtung das Auftreten von Rotationsinstabilität in radialer Richtung behindert.

Wir nehmen an, ein Volumenelement werde von seiner Ausgangslage $(r, \vartheta, \omega)$ aus um eine Strecke $\Delta r$ radial nach außen bewegt $(\vartheta=$ const) und zwar so schnell bzw. nur so weit, daß es keine Gelegenheit findet, seinen Drehimpuls dem seiner Umgebung anzugleichen. Dann unterliegt es wegen

$$
\omega r^{2} \sin ^{2} \vartheta=\mathrm{const}
$$

am neuen Ort einer Zentrifugalbeschleunigung

$$
\left(\frac{\omega r^{2}}{(r+\Delta r)^{2}}\right)^{2}(r+\Delta r) \sin \vartheta
$$


während seiner Umgebung der Wert

$$
\left(\omega+\frac{\partial \omega}{\partial r} \Delta r\right)^{2}(r+\Delta r) \sin \vartheta
$$

entspricht. Die Differenz der beiden Ausdrücke ergibt, wenn man quadratische und höhere Glieder in $\Delta r$ vernachlässigt, für den Überschuß an Zentrifugalbeschleunigung, dem das Element im Falle einer herrschenden Rotationsinstabilität unterworfen wäre

$$
2 \omega^{2} \sin \vartheta\left(2+\frac{\partial \ln \omega}{\partial \ln r}\right) \Delta r .
$$

Dann muß wenigstens die $r$-Komponente dieser Kraft durch die der thermischen Stabilität entsprechende in Richtung der Schwerkraft wirkende Beschleunigung kompensiert werden, damit indifferentes Gleichgewicht herrscht. Diese Beschleunigung ist im Falle geringer Abplattung der Niveauflächen, zu vernachlässigendem Strahlungsdruck und bei konstantem mittleren Molekulargewicht gegeben durch

$$
g \cdot \Delta \nabla \ln T \cdot \Delta r
$$

und man erhält als Bedingung für Stabilität gegen stationäre schnellere, d.h. in irgendeiner Näherung als adiabatisch anzusehende meridionale Zirkulationen, daß die Summe der beiden Arbeitsbeträge

$$
\begin{aligned}
\varrho \omega^{2} \sin ^{2} \vartheta & \left(2+\frac{\partial \ln \omega}{\partial \ln r}\right)(\Delta r)^{2}+ \\
& \frac{1}{2} \varrho g \Delta \nabla \ln T(\Delta r)^{2}>0
\end{aligned}
$$

sein $\mathrm{mu}$.

Um eine Bedingung für Stabilität gegenüber nichtstationären Bewegungen turbulenter Art zu erhalten, muß man die dabei von den scheinbaren Schubspannungen geleistete Arbeit mit in Rechnung setzen. Diese ist bei der gleichen radialen Verschiebung gegeben durch

$$
-\varrho w^{\prime} v^{\prime}(\Delta r)^{2} \text {. }
$$

Man muß nun noch eine Annahme machen über den Grad der Isotropie der turbulenten Störung. Bei vollständiger Isotropie und reiner Rotationsströmung ist

$$
v^{\prime}=w^{\prime}=\omega \sin \vartheta \sqrt{\left(\frac{\partial \ln \omega}{\partial \ln r}\right)^{2}+\left(\frac{\partial \ln \omega}{\partial \vartheta}\right)^{2}} .
$$

Es ist die positive Wurzel zu nehmen. Außerdem besitzt in diesem Fall, wie $\mathrm{Fues}^{\mathbf{1 4}}$ gezeigt hat,

14 E. Fues, Z. Physik 118, 409 [1941]. jedes Element am neuen Ort im Mittel gerade die diesem zukommende Winkelgeschwindigkeit, und die von bzw. gegen die Zentrifugalkraft zu leistende Arbeit wird daher

$$
\varrho \omega^{2} \sin ^{2} \vartheta \frac{\partial \ln \omega}{\partial \ln r}(\Delta r)^{2} .
$$

Dann lautet die Stabilitätsbedingung

$$
\begin{aligned}
& \varrho \omega^{2} \sin ^{2} \vartheta \frac{\partial \ln \omega}{\partial \ln r}(\Delta r)^{2}+\frac{1}{2} g \varrho \Delta \nabla \ln T(\Delta r)^{2} \\
& -\varrho \omega^{2} \sin ^{2} \vartheta\left[\left(\frac{\partial \ln \omega}{\partial \ln r}\right)^{2}+\left(\frac{\partial \ln \omega}{\partial \vartheta}\right)^{2}\right](\Delta r)^{2}>0 . \quad(3.4
\end{aligned}
$$

Dagegen berechnet sich bei einer rein anisotropen Störung in radialer Richtung die Arbeit gegen die Zentrifugalkraft wieder wie in (3.2), und man hat für $v^{\prime}$ den Geschwindigkeitsunterschied einzusetzen, den das Element gegenüber seiner Umgebung am neuen Ort aufweist, wenn es seinen Drehimpuls beibehalten hat. D. h.

$$
\begin{aligned}
& v^{\prime}=\omega \sin \vartheta\left(2+\frac{\partial \ln \omega}{\partial \ln r}\right) \\
& w^{\prime}=\omega \sin \vartheta \frac{\partial \ln \omega}{\partial \ln r},
\end{aligned}
$$

und $\quad w^{\prime}=\omega \sin \vartheta \frac{\partial \ln \omega}{\partial \ln r}$,

dann lautet die Stabilitätsbedingung

$$
\begin{aligned}
& \varrho \omega^{2} \sin ^{2} \vartheta\left(2+\frac{\partial \ln \omega}{\partial \ln r}\right)(\Delta r)^{2}+\frac{1}{2} g \varrho \Delta \nabla \ln T(\Delta r)^{2} \\
& -\varrho \omega^{2} \sin ^{2} \vartheta\left(2+\frac{\partial \ln \omega}{\partial \ln r}\right) \frac{\partial \ln \omega}{\partial \ln r}(\Delta r)^{2}>0 . \quad(3.6)
\end{aligned}
$$

Sofern $\partial \omega / \partial r \leqq 0$ ist, hat man demnach als Bedingung für:

1. Stabilität gegen schnellere meridionale Zirkulationen aus (3.2)

$$
\frac{\partial \ln \omega}{\partial \ln r}>-2-\frac{1}{2} g \frac{\Delta \nabla \ln T}{\omega^{2} \sin ^{2} \vartheta},
$$

2. Stabilität gegen anisotrope Turbulenz mit bevorzugt radialer Richtung aus (3.6)

$$
\frac{\partial \ln \omega}{\partial \ln r}>-\frac{1}{2}-\sqrt{\frac{9}{4}+\frac{1}{2} g \frac{\Delta \nabla \ln T}{\omega^{2} \sin ^{2} \vartheta}},
$$

3. Stabilität gegen isotrope Turbulenz aus (3.4)

$$
\frac{\partial \ln \omega}{\partial \ln r}>\frac{1}{2}-\sqrt{\frac{1}{4}+\frac{1}{2} g \frac{\Delta \nabla \ln T}{\omega^{2} \sin ^{2} \vartheta}-\left(\frac{\partial \ln \omega}{\partial \vartheta}\right)^{2}} .
$$

Für indifferente thermische Schichtung $(\Delta \nabla \ln T=0$ ) werden die Kriterien (3.7) und (3.8) identisch mit einem schon von $\mathrm{R}$ a y leig $\mathrm{h}^{15}$ ange-

${ }^{15}$ Lord Rayleigh, Proc. Roy. Soc. [London], Ser. A 93, 148 [1917]. 
gebenen Kriterium, wonach Rotationsstabilität herrscht, solange der Drehimpuls mit größer werdender Entfernung von der Rotationsachse zunimmt, d. h. für

$\frac{\partial \ln \omega}{\partial \ln s}=\frac{\partial \ln \omega}{\partial \ln r} \sin ^{2} \vartheta+\frac{\partial \ln \omega}{\partial \vartheta} \sin \vartheta \cos \vartheta>-2$.

Insofern wären die Kriterien (3.7) und (3.8) noch zu ergänzen durch die Bedingung

$$
\frac{\partial \ln \omega}{\partial \vartheta}>-2 \operatorname{ctg} \vartheta
$$

Welches der drei genannten Kriterien nun im gegebenen Fall anzuwenden ist, läßt sich streng nicht entscheiden ${ }^{16}$. Um aber einen gewissen Anhalt zu haben, kann man eine $R$ e y nold s sche Zahl definieren, indem man das Verhältnis der turbulenten Viskosität $\eta_{T}$ zur molekularen Viskosität $\eta$ bildet.

Indem man in

$$
\operatorname{Re}=\frac{\eta_{T}}{\eta} .
$$

$$
\eta_{T}=\varrho w l
$$

für die durch Rotationsinstabilität entstehende Turbulenzgeschwindigkeit entsprechend (3.3)

$$
w=\omega l \sin \vartheta \sqrt{\left(\frac{\partial \ln \omega}{\partial \ln r}\right)^{2}+\left(\frac{\partial \ln \omega}{\partial \vartheta}\right)^{2}}
$$

und für die Lineardimension $l$ die homogene Dicke der Schicht ${ }^{17}$

einsetzt, wird

$$
h=\frac{\Re T}{\mu g}
$$

$$
\eta_{T} \approx \varrho \omega h^{2} \sin \vartheta \sqrt{\left(\frac{\partial \ln \omega}{\partial \ln r}\right)^{2}+\left(\frac{\partial \ln \omega}{\partial \vartheta}\right)^{2}}
$$

Da die Reynoldssche Zahl für einen Zustand gelten soll, in dem die Turbulenż auch aufrecht erhalten bleibt und nicht durch die erhöhte Vis-

16 Historisch gesehen ist die Stabilitätsbedingung (3.2) der Spezialfall eines von Solbergica und von $H ø$ il and ${ }^{16 b}$ angegebenen Kriteriums, insofern hier nur die radiale Verrückung betrachtet wird. Sie ist aber auch die Verallgemeinerung eines von Randersi2a und Biermann ${ }^{16 c}$ für den Fall starrer Rotation aufgestellten Kriteriums. Die Stabilitätsbedingung (3.6) erhält man, wenn man zwei von Prand t1 ${ }^{16 d}$ bereits 1929 angegebene Kriterien sinngemäß kombiniert [1. c. ${ }^{16 d}$ Gl. (1) und (6)]. Sieht man dabei dann von der durch die scheinbaren Schubspannungen geleisteten Arbeit ab, so entsteht wieder das Kriterium (3.2). Dabei ist zu beachten, daß die von Prandtl [1. c. ${ }^{16 d} \mathrm{Gl}$. (5)] durchgeführte Mittelung bei Annahme von Isotropie nicht mehr zulässig ist. Die Aufdeckung dieses Umstandes durch Fues ${ }^{14}$ Wasiutynsi ${ }^{16 e}$ und Biermann führte damit zu der Stabilitätsbedingung (3.4). kosität wieder zum Verschwinden gebracht wird, muß man in (3.13) den von der äußeren Kraft erzwungenen Gradienten z. B. (3.1) einsetzen und erhält so für die äußeren Teile der Sonne

$$
\operatorname{Re} \approx 10^{0,22} \frac{h}{\eta}(\sin \vartheta)^{\eta / 8} \sqrt{\varrho \omega_{0}\left(\frac{R}{r}\right)^{3}} .
$$

Nach Experimenten, bei denen sich ein Medium, z. B. Wasser, zwischen zwei koaxialen mit einzeln variierbarer Winkelgeschwindigkeit rotierenden Zylindern befindet, erhält man bei Rotationsinstabilität stationäre dreidimensionale Strömungsformen bis zu R e y n old s schen Zahlen der Größenordnung $10^{4}$ bis $10^{5}$. Bei hinreichend großer Instabilität und höheren Werten erfolgt der Übergang in eine nichtstationäre turbulente Bewegung, die aber immer noch vorwiegend anisotrop zu sein scheint. Als Anzeichen für einen merkbaren isotropen Anteil müßte nach (3.9) gelten, daß die Stabilitätsgrenze sich mehr nach dem Falle starrer Rotation zu verschiebt. Bis zu der höchsten bisher erreichten Reynoldsschen Zahl von der Größenordnung $10^{5}$ konnte aber nichts derartiges bemerkt werden, so daß mit vorwiegend isotroper Turbulenz und daher mit dem Kriterium (3.9) wohl erst bei außerordentlich hohen R e y n old s schen Zahlen zu rechnen sein wird.

Als Modell für die Druck-, Temperatur- und Dichteverteilung in den äußeren Teilen der Sonne sei von zwei Rechnúngen von Biermann ${ }^{18}$ ausgegangen, die auf den beiden extremen Annahmen beruhen, daß einmal der Energietransport ausschließlich durch Strahlung erfolge (S-Lösung), zum andern $\mathrm{da} ß$ er vollständig durch konvektive Leitung besorgt werde (K-Lösung). Für das eigentliche Innere werde eine Rechnung von $\mathrm{Keller}{ }^{19}$ zugrunde gelegt, die auf der Annahme beruht,

16a H. Solberg, Le mouvement d'inertie de l'atmosphère stable et son rôle dans la théorie des cyclones (Reprint 1936).

16b E. Høiland, Avhandl. utg. av Det Norske Videnskaps-Akademi i Oslo (I. Mat. Naturv. Klasse Nr. 11, 1941).

16 c L. B i erm a n n, Z. Astrophysik 25, 135 [1948]. 16d L. Prandtl, Vorträge aus dem Gebiet der Aerodynamik, Aachen 1929; J. Springer, Berlin.

16e J. Wasiutynski, Astrophysica Norvegica IV p. $47 \mathrm{ff}$. [1946].

16 f L. B i e rm a n n, Z. Astrophysik 28, 304 [1951].

17 L. B i erm a n n, Z. Astrophysik 25, 161 [1948].

18 L. Bierma n n, Z. Astrophysik 21, 320 [1942].

19 G. K ell e r, Dissertation Columbia Univ. 1948. Auszug daraus: Astrophysic. J. 108, 347 [1948]. 
daß sich die Sonne bis auf einen die Energiequellen umfassenden konvektiven Kern im Strahlungsgleichgewicht befindet, und außerdem voraussetzt, daß unter den schweren Elementen der Sauerstoff absolut überwiegt (O-Modell). Der Druck- und Temperaturverlauf ist in Abb. 1 in

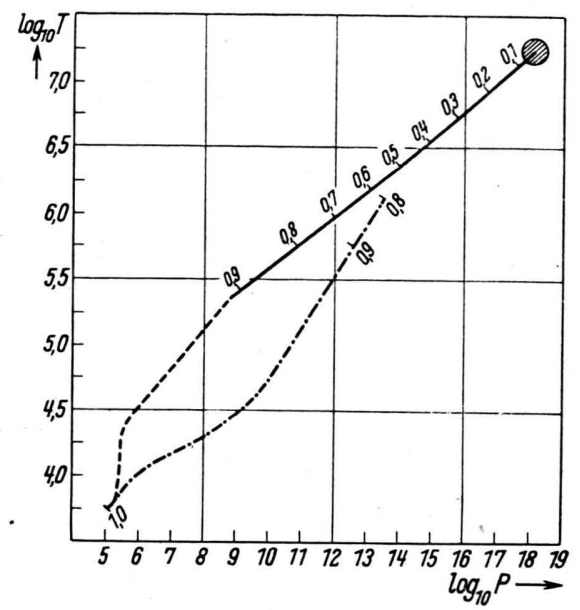

Abb. 1. Druck- und Temperaturverlauf im Innern der Sonne.

- O-Modell n. K ell er ${ }^{19}$; . . . . . . S-Lösung, -............ K-Lösung, beide nach Bier$\operatorname{mann}{ }^{16 f}$.

Auf den Kurven ist der relative Radius $r / R$ angegeben.

Form eines Wegdiagrammes wiedergegeben. Um die S-Lösung an das O-Modell etwas anzupassen, wurde vom Fuße der WKZ an willkürlich mit einem konstanten Gradienten $\mathrm{d} \ln T / \mathrm{d} \ln P=0,30$ gerechnet.

In Kellers Rechnung, die für die chemische Zusammensetzung der Sonne zu dem unwahrscheinlichen Ergebnis $Y=0$ führte, ging eine Unsicherheit bezüglich der Lebensdauer des ${ }^{12} \mathrm{C}$ Kernes ein, die erst bei Abschluß seiner Arbeit bekannt wurde und daher nicht mehr berücksichtigt werden konnte. Da neuere Untersuchungen ${ }^{20}$ aber sowieso für eine niedrigere Mittelpunktstemperatur der Sonne zu sprechen scheinen, wodurch die H-H-Reaktion sogar höhere Chancen gegenüber dem Kohlenstoffzyklus bekäme, erscheint ein Versuch, die Konsistenz der hier verwendeten Modelle zu verbessern, im Augenblick nicht angebracht. Die erhaltenen Ergebnisse sind

20 I. Epstein, Astrophysic. J. 112, 207 [1950].

21 c.f.Ch apm an-Cowling, Mathematical Theo- daher in numerischer Hinsicht mit einigem Vorbehalt aufzunehmen. Wahrscheinlich wird man in Zukunft mit im allgemeinen etwas niedrigeren Temperaturen zu rechnen haben.

Mit Hilfe dieser Modellvorstellungen werde nun die molekulare innere Reibung und die Strahlungsviskosität berechnet. Der Viskositätskoeffizient für ein ionisiertes einfaches Gas ist 21

$$
\eta_{M}=1,15 \frac{5}{16}\left(\frac{m k T}{\pi}\right)^{\frac{1}{2}}\left(\frac{2 k T}{Z^{2} e^{2}}\right)^{2} / A_{2}(2)
$$
mit

$$
A_{2}(2)=2\left\{\ln \left(1+v_{01}^{2}\right)-\frac{v_{01}^{2}}{1+v_{01}^{2}}\right\} .
$$

Dabei wird mit einer abstoßenden Coulomb-Kraft gerechnet und außerdem nur mit Zweierstößen. Zur Definition des Abschneideradius $v_{01}$ wird der mittlere Abstand $d$ zwischen zwei Molekülen benützt.

$$
v_{01}=\frac{4 k T}{Z^{2} e^{2}} d ; \quad d \approx n^{-\frac{1}{3}} .
$$

Es läßt sich zeigen, daß bei der chemischen $\mathrm{Zu}$ sammensetzung des O-Modelles der Wasserstoff für die Viskosität ausschlaggebend ist. Durch die freien Elektronen wird der mittlere Impuls verkleinert und damit die Viskosität des Wasserstoffgases entsprechend $\left(m_{\mathrm{e}} / m_{\mathrm{H}}\right)^{1 / 2}$ um ca. $2 \%$ herabgesetzt. Einem Massenanteil von 0,03 entsprechen zahlenmäßig 1,9\% Sauerstoffatome, deren Wechselwirkung mit den Wasserstoffkernen um $Z^{2} A^{1 / 2}=264$-mal größer ist als die der Protonen untereinander, wodurch die Viskosität um ca. $50 \%$ erhöht wird. Die genaue Rechnung gibt $46,5 \%$. Die Größe $A_{2}(2)$ ändert sich in der Sonne nur wenig und kann praktisch als konstant angenommen werden. Für das O-Modell ist $A_{2}(2)=17,0 \pm$ 2,5 , so daß man insgesamt für (3.17) erhält

$$
\eta_{M}=10^{-15,4} T^{5 / 2} \text {. }
$$

Der Koeffizient der Strahlungsviskosität ist nach $\mathrm{Jeans}^{9}$ (vgl. auch Klauder ${ }^{22}$ )

$$
\eta_{R}=\frac{2}{15} \frac{a T^{4}}{c \varkappa \varrho} \text {. }
$$

Das O-Modell beruht auf folgendem Ansatz für den Absorptionskoeffizienten

$$
\begin{aligned}
& \varkappa=\varkappa_{0} \varrho^{0,8} T^{-4,5} \text { mit } \\
& \varkappa_{0}=1,70 \cdot 10^{31}(1-X-Y)(1+X)^{0,8},
\end{aligned}
$$

rie of Nonuniform Gases, University Press, Cambridge 1939 p. 179.

22 H. K la u d e r, Astronom. Nachr. 273, 190 [1942]. 
worin ein empirischer Ausdruck für den Guillotinefaktor enthalten ist. Mit $X=0,97$ und $Y=0$ erhält man damit für (3.21)

$$
\eta_{R}=10^{-55,4} T^{8,5} \varrho^{-1,8} .
$$

Sowohl in (3.20) wie in (3.23) dürfte die Unsicherheit in der Temperatur mehr ausmachen als der Fehler in der chemischen Zusammensetzung.

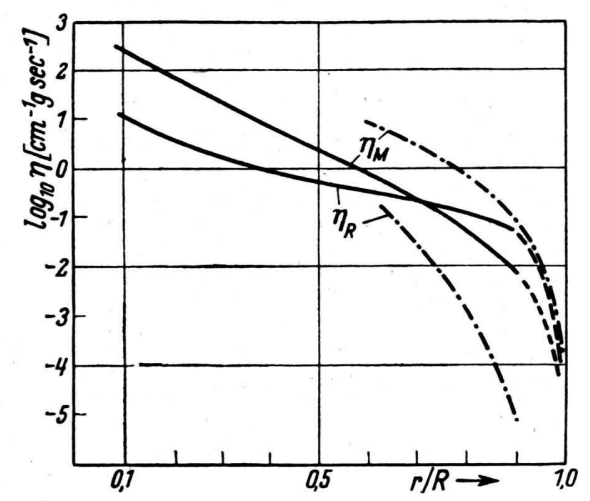

Abb. 2. Koeffizient der molekularen Viskosität und der Strahlungsviskosität (Bezeichnung der Modelle wie in Abb. 1).

Die so berechneten Werte von $\eta_{M}$ und $\eta_{R}$ sind in Abb. 2 wiedergegeben. Daß für das O-Modell die Strahlungsviskosität in den äußeren Teilen überwiegt, ist eine nicht ernst zu nehmende Besonderheit dieses Modelles, die wesentlich auf der außerordentlichen Dichtekonzentration beruht (der mittlere Polytropenindex ist $n=3,9$ !). Soweit die beiden Viskositätskoeffizienten von der gleichen Größenordnung sind, werde im folgenden unter $\eta$ stets die Summe $\eta=\eta_{M}+\eta_{R}$ verstanden.

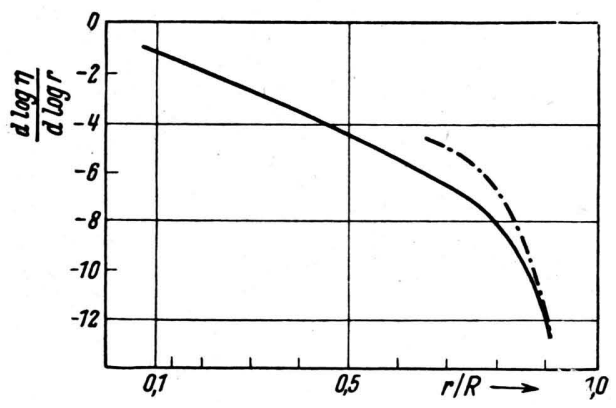

Abb. 3. Der Gradient der Viskosität $\eta=\eta_{M}+\eta_{R}$.

Abb. 3 gibt den Verlauf von $d \ln \eta / d \ln r$, und ein Vergleich mit (2.1) zeigt, daß dieses Glied keinesfalls vernachlässigt werden kann, sofern die Vis- kosität überhaupt eine Rolle spielt. Die kinematische Zähigkeit, Abb. 4, zeigt naturgemäß ein sehr stark von der Wahl des Modelles, d. h. von

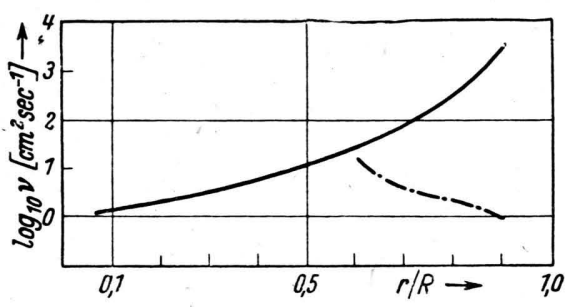

Abb. 4. Kinematische Zähigkeit $v=\eta / \varrho$.

der Temperatur- und Dichteverteilung abhängendes Verhalten. - Der K-Lösung entspricht in den äußeren Teilen eine um ca. drei Zehnerpotenzen höhere Dichte; dies hat die Abnahme der kinematischen Zähigkeit (welche hier die Dichte im Nenner enthält) nach außen zur Folge, und bewirkt außerdem, daß weder die Viskosität noch der Strahlungsstrom praktisch Einfluß auf die Verteilung der Winkelgeschwindigkeit hat. Für die S-Lösung und das O-Modell ergibt sich dagegen (s. Abb. 5), daß der Strahlungsstrom allein bis in eine Tiefe von etwa 0,85 im relativen Radius schon imstande ist, das Rotationsgesetz zu bestimmen.

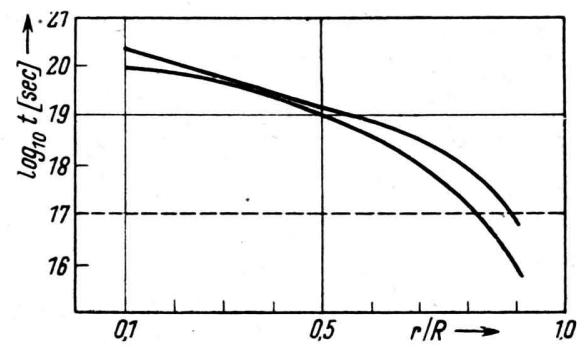

Abb. 5. Die Zeitskala, in der die Viskosität (obere Kurve) und der Strahlungsstrom (untere Kurve) die Verteilung der Winkelgeschwindigkeit bestimmen.

Um nun zu entscheiden, ob eine äußere $A b$ bremsung von dem abgeschätzten Betrag tatsächlich die Existenz einer rotationsinstabilen Zone zur Folge hat, müssen zunächst folgende Fragen beantwortet werden: 1. Wie tief reicht die Wasserstoffkonvektionszone? -2 . Von welcher Tiefe an würde die molekulare Viskosität imstande sein, den geforderten Drehimpulsstrom zu bewirken, ohne daß die Schichtung dabei rotationsinstabil wird ? - 3. Bis in welche Tiefe reicht der Einfluß der äußeren Abbremsung? 


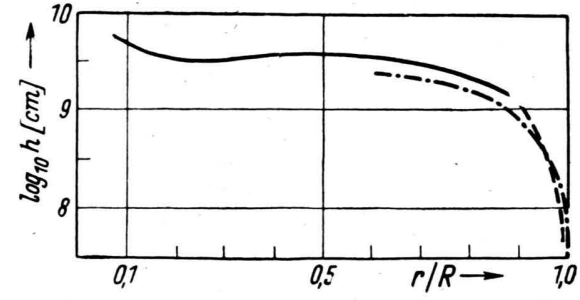

Abb. 6. Die homogene Dicke.

Bezeichnet man die entsprechenden Tiefen unter der Oberfläche, $d=R-r$, mit $d_{1}, d_{2}$ und $d_{3}$, so ist

$$
d_{3} \geqq d_{2}>d_{1} \text { bzw. } d_{2} \geqq d_{3}>d_{1}
$$

eine notwendige Bedingung für die Existenz einer rotationsinstabilen Zone. Die dritte Frage wird sich dabei erst nach der zweiten beantworten lassen, da man, um den in $d_{3}$ enthaltenen Gesamtdrehimpuls zu berechnen, das Rotationsgesetz in dieser Zone kennen muß.

Die Dicke der WKZ ist im Falle der S-Lösung äußerst gering und beträgt nur $d_{1 \mathrm{~s}}=400 \mathrm{~km}$. Im Falle der K-Lösung beträgt die Tiefe des Ionisationsbereiches des Wasserstoffes $5000 \mathrm{~km}$. An seiner inneren Grenze ist die Schichtung jedoch noch sehr instabil, weil der zum Strahlungstransport der Energie erforderliche Temperaturgradient infolge der hohen Opazität groß gegen den adiabatischen Temperaturgradienten geworden ist. Die Instabilität kann daher bis tief ins Innere reichen, ohne daß sich genau sagen ließe, wie tief. Jedenfalls muß man mit einer Dicke der thermisch instabilen Zone von $d_{1 \mathrm{~K}}=10000$ bis $100000 \mathrm{~km}$ rechnen.

Um die zweite Frage zu beantworten, muß zuerst entschieden werden, mit welchem Stabilitätskriterium man zu rechnen hat. Berechnet man etwa für die außerhalb $r=0,8 R$ gelegenen Teile der Sonne die Reynoldssche Zahl nach (3.16), so findet man für die S-Lösung und das anschließende O-Modell Werte der Größenordnung $10^{6}$, für die K-Lösung dagegen etwas höhere Werte, die von $10^{8}$ am Fuße der Wasserstoffionisationszone bis auf $10^{7}$ abnehmen. Trotzdem wird man nach dem oben Gesagten (S.111) in beiden Fällen wohl mit dem Kriterium (3.8) für anisotrope Turbulenz zu rechnen haben. Setzt man demzufolge (3.1) in (3.8) ein, so erhält man eine Zone, in der Rotationsinstabilität herrschen muß, sofern der Drehimpulstransport nicht durch eine auf thermischer Instabilität beruhende Konvektion er- folgt und der Gültigkeitsbereich von (3.1) tatsächlich bis in diese Tiefe reicht. Die Dicke dieser Zone nimmt nach den Polen zu ab (s. Abb. 7 und 8) und beträgt am Äquator für die S-Lösung $d_{2 \mathrm{~S}}=$ $23000 \mathrm{~km}$ und für die K-Lösung $d_{2 \mathrm{~K}}=147000$ $\mathrm{km}$.

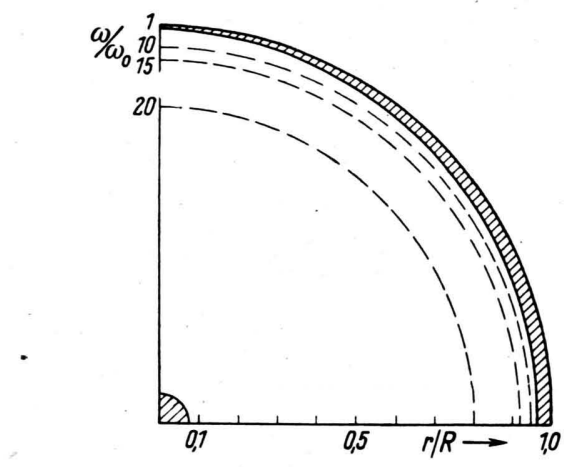

Abb. 7. Die rotationsinstabile Zone im Falle der S-Lösung.

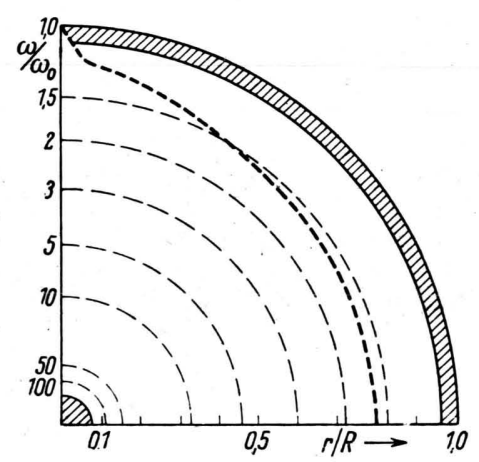

Abb. 8. Die rotationsinstabile Zone im Falle der K-Lösung.

Das Rotationsgesetz wird in der WKZ je nach dem Grad der Isotropie bzw. Anisotropie der herrschenden Turbulenz zwischen dem Fall einer praktisch starren Rotation und dem eines weitgehend ausgeglichenen Rotationsmomentes liegen. In der rotationsinstabilen Zone wird man dagegen entsprechend dem angenommenen Stabilitätskriterium nur mit dem letzteren Fall, d. h. mit $\partial \ln \omega / \partial \ln r=-2, \mathrm{zu}$ rechnen haben. In den stabil geschichteten Teilen schließlich wird das Rotationsgesetz nach (3.1) durch die molekulare Viskosität bestimmt. Das ergibt für die S-Lösung zunächst einen außerordentlich starken Gradienten der Winkelgeschwindigkeit $(\partial \ln \omega / \partial \ln r \approx-600)$, der mit zunehmender Winkelgeschwindigkeit nach innen rasch schwächer wird, so daß bei 
$r=0,85 R$ der oben erwähnte Einfluß des Strahlungsstromes gerade noch von etwa der gleichen Größenordnung ist. Insgesamt strebt die Winkelgeschwindigkeit demnach einem Grenzwert zu, der etwa das 20-fache des an der Oberfläche beobachteten Wertes beträgt. Dabei ist zu beachten, daß sich einmal die erhaltene Zone radialer Instabilität nach den Polen zu stark verjüngt (Abb. 7), und daß außerdem in diesen Teilen der Sonne ein sehr großer Gradient der molekularen Viskosität herrscht (Abb. 3). Das aber hat zur Folge, daß sich bei der Rechnung in höheren heliographischen Breiten eine sehr viel stärkere Zunahme der Winkelgeschwindigkeit im Radius nách innen ergibt als am Äquator und dadurch die Stabilitätsbedingung (3.11) in Richtung $\vartheta$ verletzt wird. Das Ergebnis ist zwar rein formal die Folge der willkürlichen Aufspaltung der Stabilitätsrechnung in zwei unabhängige Bedingungen, es bedeutet aber, daß ein starker Gradient der Viskosität in diesem Falle eine annähernd kugelsymmetrische Verteilung der Winkelgeschwindigkeit bewirken wird. Für die K-Lösung würde die angenommene äußere Abbremsung in einer Tiefe größer als $d_{2 \mathrm{~K}}$ bei stabiler Schichtung keine nennenswerte weitere Zunahme der Winkelgeschwindigkeit nach innen ergeben.

Der Bereich, auf den sich die äußere Abbremsung erstreckt und in dem (3.1) gilt, ist, wie schon gesagt, dadurch eingeschränkt, daß der in ihm enthaltene Gesamtdrehimpuls nicht größer als etwa $10^{45,3} \mathrm{~cm}^{2} \mathrm{~g} \mathrm{sec}^{-1}$ sein darf, d. h.

$\frac{2}{5} \bar{\omega} \frac{4 \pi}{3} R^{5}\left(1-x^{5}\right) \bar{\varrho} \leqq 10^{45,3} \mathrm{~cm}^{2} \mathrm{~g} \mathrm{sec}^{-1}$.

Dabei bezeichnet $\bar{\omega}$ die mittlere Winkelgeschwindigkeit und $\bar{\varrho}$ die mittlere Dichte in dem gesuchten Bereich und $x=r / R$ seine innere Grenze. Die Masse der außerhalb $r$ liegenden Teile läßt sich in hinreichender Näherung durch den Druck ausdrücken,

$$
P \approx \frac{4 \pi}{3} R^{3}\left(1-x^{3}\right) \frac{g \bar{\varrho}}{4 \pi r^{2}}
$$

und, indem man $\bar{\varrho}$ eliminiert, erhält man aus (3.25) und (3.26)

$$
x^{4} \frac{1-x^{5}}{1-x^{3}} \frac{\bar{\omega}}{\omega_{0}} P \leqq 10^{11,22} \mathrm{dyn} / \mathrm{cm}^{2} .
$$

Aus dieser Bedingung ergibt sich (vgl. Abb. 1) bei Berücksichtigung des entsprechenden Rotationsgesetzes die Tiefe $d=R(1-x)$ für die S-Lösụng zu $d_{3 \mathrm{~s}}=140000 \mathrm{~km}$ und für die K-Lösung zu $d_{3 \mathrm{~K}}=21000 \mathrm{~km}$. Insgesamt hat man demnach für die

\begin{tabular}{l|c|c}
\multicolumn{1}{c}{} & \multicolumn{1}{c}{ S-Lösung } & K-Lösung \\
\cline { 2 - 3 }$d_{1}$ & $400 \mathrm{~km}$ & $10000-100000 \mathrm{~km}$ \\
$d_{2}$ & 23000 & 147000 \\
$d_{3}$ & 140000 & 21000
\end{tabular}

Die Bedingung (3.24) für die Existenz einer rotationsinstabilen Zone ist somit nur im Falle der S-Lösung eindeutig erfüllt; bei der K-Lösung dürfte die Dicke der thermisch instabilen Zone die angegebene untere Grenze von $10000 \mathrm{~km}$ nicht überschreiten, wenn die Bedingung erfüllt sein soll.

Während für die K-Lösung wegen $\Delta \nabla \ln T \approx 0$ die Kriterien für meridionale Zirkulationen (3.7) und für anisotrope Turbulenz (3.8) identisch sind, werden für die S-Lösung wegen der bei reinem Strahlungstransport der Energie außerordentlich hohen thermischen Stabilität praktisch die Kriterien (3.8) und (3.9) einander gleich, und das Kriterium (3.7) für meridionale Zirkulationen würde überhaupt keine rotationsinstabile Zone ergeben.

Die Erwartung, daß eine äußere Abbremsung der Sonne das Auftreten meridionaler Zirkulationen im Sinne der Bjerknesschen Vorstellung zur Folge hat, scheint demnach zwar in keinem der beiden diskutierten Fälle befriedigend erfüllt; bedenkt man aber, daß beide Modelle in einem gewissen Sinn Grenzfälle sind, so erscheint es keineswegs ausgeschlossen, daß sich - falls die Wirklichkeit zwischen diesen Lösungen liegt - das Sonnenfleckenphänomen doch in der angegebenen Weise hydrodynamisch ausreichend begründen läßt.

\section{Das Rotationsgesetz an der Oberfläche}

Das Sonnenfleckenphänomen

Bevor die Diskussion des Bjerknesschen Modelles fortgeführt wird, sei noch auf einen $\mathrm{Zu}$ sammenhang hingewiesen, der sich, wie bereits Randers ${ }^{6 \mathrm{~b}}$ bemerkt hat, zwischen drei an der Oberfläche unmittelbar beobachtbaren Erscheinungen ergibt und einige weitere wichtige Schlüsse erlaubt. Die drei Phänomene sind: die Abnahme der Winkelgeschwindigkeit nach den Polen zu, die Turbulenz in der WKZ, soweit sie sich in der 
Granulation bemerkbar macht, und die äquatorwärts gerichtete Wanderung der Sonnenfleckenzone innerhalb des Spörerschen Zyklus.

Das Rotationsgesetz an der Oberfläche lautet nach Greenwicher Beobachtungen an langlebigen Sonnenflecken für $\vartheta>55^{0}$

$$
\omega(\vartheta)=\omega_{0}\left(1-0,18 \cos ^{2} \vartheta\right) .
$$

Für das Spektrum der Turbulenz in der WKZ ergaben Untersuchungen von $\mathrm{Rich}$ ards on und $\mathrm{Schwarzschild}{ }^{23}$ sowie von $\mathrm{Su} \mathrm{Shu} \mathrm{Hu}$ a $\mathrm{g}^{24}$, daß allem Anschein nach den kleineren Turbulenzelementen die größere Geschwindigkeit zukommt und man in einem Bereich von $l=$ $100 \mathrm{~km}$ bis $l=1000 \mathrm{~km}$ für die kinematische Zähigkeit mit dem annähernd konstanten Wert

$$
v_{0}=w l \approx 10^{12,3} \mathrm{~cm}^{2} / \mathrm{sec}
$$

rechnen kann. Das entspricht einer turbulenten Viskosität $\eta_{T} \approx 10^{6} \mathrm{~cm}^{-1} \mathrm{~g} \mathrm{sec}-1$ und damit einer Reynoldsschen Zahl der Größenordnung $10^{12}$.

Der infolge der turbulenten Viskosität und des beobachteten Gradienten der Winkelgeschwindigkeit polwärts fließende Drehimpulsstrom müßte jedoch die bestehenden Unterschiede in der Winkelgeschwindigkeit in größenordnungsmäßig $R^{2} / v_{0}=10^{9,4} \mathrm{sec}$, das ist in rund 80 Jahren, ausgeglichen haben, wenn er nicht durch einen entgegengesetzt gerichteten Materiestrom kompensiert würde. Für dessen Geschwindigkeit erhält man, indem man in (1.11) für $\left[I_{\vartheta}\right]_{0}=0$ annimmt, entsprechend (4.1) und (4.2)

$$
\overline{\left[v_{\vartheta}\right]_{0}}=\frac{v_{0}}{R} \frac{\partial \ln \omega}{\partial \vartheta} \approx 10^{0,8} \mathrm{~cm} / \mathrm{sec} .
$$

Der Wanderung der Sonnenfleckenzone, die sich in 11 Jahren um ca. $30^{\circ}$ in heliographischer Breite nach dem Äquator zu verschiebt, würde, wenn man sie als eine materielle Strömung auffaßt, eine Geschwindigkeit von im Mittel $\overline{\left[v_{\vartheta}\right]_{0}}=$ $10^{2} \mathrm{~cm} / \mathrm{sec}$ entsprechen. Das ist etwa eine Zehnerpotenz mehr als der theoretische Wert (4.3). Um den Unterschied zu erklären, könnte man einmal annehmen, daß die kinematische Zähigkeit der WKZ in Wirklichkeit entsprechend auch um eine Größenordnung höher ist, es kann aber auch der Umstand dafür verantwortlich gemacht werden, daß die Turbulenz in der WKZ kaum völlig isotrop sein wird ${ }^{24}$. Da ein anisotroper Austausch im Gegensatz zur molekularen Viskosität und einer

23 R. S. Richardson u. M. Schwarzschild, Astrophysic. J. 111, 351 [1950]. völlig isotropen Turbulenz aber nicht auf einen Ausgleich der Winkelgeschwindigkeit hinwirkt, sondern auf einen Ausgleich des Rotationsmomentes in einer bestimmten Richtung, kann man dieser zusätzlichen Möglichkeit dadurch Rechnung tragen, daß man außer der Größe $A \equiv \eta_{T}$ für den isotropen Austausch die Austauschgrößen $A_{r}$ und $A_{\vartheta}$ für die anisotropen Anteile definiert und dementsprechend in den Gl. (1.10) und (1.11) für den Drehimpulsstrom die Glieder

$$
-A_{r} \frac{\partial}{\partial r}\left(\omega r^{2} \sin ^{2} \vartheta\right) \quad \text { bzw. } \quad-\mathrm{A}_{\vartheta} \frac{1}{r} \frac{\partial}{\partial \vartheta}\left(\omega r^{2} \sin ^{2} \vartheta\right)
$$

hinzufügt. Dann erhält man an Stelle von (4.3)

$$
\left[v_{\vartheta}\right]_{0}=\frac{A+A_{\vartheta}}{\varrho_{0} R} \frac{\partial \ln \omega}{\partial \vartheta}+\frac{2 A_{\vartheta}}{\varrho_{0} R} \operatorname{ctg} \vartheta .
$$

Dies würde für den Fall, daß der anisotrope Austauschkoeffizient etwa von der gleichen Größenordnung ist wie der isotrope $\left(A_{\vartheta} \approx A\right)$, auf Grund des Wertes (4.2) für $A / \varrho_{0}$ und für $\vartheta=45^{0}$ eine Geschwindigkeit $\overline{\left[v_{\vartheta}\right]_{0}} \approx 10^{1,9} \mathrm{~cm} / \mathrm{sec}$ ergeben.

Man wird daher als ziemlich gesichert annehmen können, daß der beobachteten Wanderung der Fleckenzone eine meridionale Strömung entspricht, deren Geschwindigkeit an der Oberfläche etwa $1 \mathrm{~m} / \mathrm{sec}$ beträgt. Diese Zirkulation muß sich aber irgendwie im Innern der Sonne schließen. Nimmt man an, daß die äquatorwärts gerichtete Strömung ausschließlich in der WKZ verläuft, und die Rückströmung über das ganze Sonneninnere erfolgt, dann läßt sich die mittlere Geschwindigkeit der letzteren nach folgendem einfachen Ansatz abschätzen,

$$
\bar{v} \approx \overline{\left[v_{\vartheta}\right]_{0}} \frac{\pi R^{2}\left(1-x_{1}^{2}\right) \bar{\varrho}_{1}}{\pi R^{2} \varrho m} .
$$

\begin{tabular}{|c|c|c|}
\hline & S-Lösung & K-Lösung \\
\hline$d_{1}$ & $400-10000 \mathrm{~km}$ & $10000-100000 \mathrm{~km}$ \\
\hline $\bar{v}$ & $10^{-7,5}-10^{-5,8}$ & $10^{-2,7}-10^{-0,55} \mathrm{~cm} / \mathrm{sec}$ \\
\hline$R / v$ & $10^{18,3}-10^{16,7}$ & $10^{13,5}-10^{11,4} \mathrm{sec}$ \\
\hline
\end{tabular}

Dabei ist $\bar{\varrho}$ die mittlere Dichte in der WKZ und $\varrho_{m}$ die mittlere Dichte im übrigen Stern. Mit $d_{1}=R\left(1-x_{1}\right)$ erhält man daraus für die

Wenn man annimmt, daß auch im Falle der SLösung die an der Sonnenoberfläche beobachtete

24 Su Shu Huang, Astrophysic. J. 112, 418 $[1950]$. 
meridionale Strömung eine Schicht von mindestens $10000 \mathrm{~km}$ Dicke umfaßt, so ist die Zeitskala $R / \bar{v}$ der Strömung im Sonneninnern in allen Fällen kleiner als das Alter der Sonne $\left(10^{17} \mathrm{sec}\right)$. D. h., eine solche Zirkulation müßte eine gleichmäßige chemische Zusammensetzung der ganzen Sonne bewirken. Andererseits wird in der Sonnenatmosphäre ein bestimmter Gehalt der Elemente Lithium und Beryllium beobachtet, die im tieferen Innern bei Temperaturen $>4 \cdot 10^{6}$ Grad sehr rasch würden umgewandelt werden ${ }^{25}$, wodurch jeder intensive Massenaustausch, sei es durch Konvektion oder durch Zirkulation, zwischen der Atmosphäre und dem tieferen Innern der Sonne ausgeschlossen erscheint. Man wird daher ebenfalls als gesichert annehmen können, daß die an der Oberfläche beobachtete meridionale Zirkulation sich nicht über das ganze Sonneninnere schließen wird, sondern auf eine äußere Zone beschränkt bleiben muß.

Für das Sonnenfleckenphänomen kann nun folgendes in den wesentlichen. Zügen der Bjerknesschen Vorstellung entsprechende Modell wenigstens qualitativ als einigerma ßen begründet gelten :

Infolge einer äußeren Abbremsung der Sonne durch das sie umgebende interplanetarische Medium wird sich an die WKZ eine rotationsinstabile Zone anschließen, in der der geforderte Drehimpulstransport durch kleinräumige meridionale Zirkulationen (Wirbelringe) geleistet wird.

Wieviele solcher Wirbelringe in der rotationsinstabilen Zone einer Hemisphäre liegen, ob nur zwei wie bei Bjerknes oder mehrere, wie man nach den Taylorschen Versuchen erwarten sollte, ist zunächst noch eine offene Frage, da dies u. a. von den Dimensionen der einzelnen Wirbel im Verhältnis zu den Ausmaßen der rotationsinstabilen Zone abhängen wird, und man noch nicht in der Lage ist zu entscheiden, ob und in welcher Weise der Durchmesser eines solchen Sonnenfleckenwirbels durch die Dicke der rotationsinstabilen Zone, durch die homogene Schichtdicke (d. i. durch den Druckgradienten und die Temperatur) oder durch den Druck selbst speziell den magnetischen Druck in seinem Innern bestimmt wird. Fest steht aber, daß je zwei aufeinanderfolgende Wirbel entgegengesetzten Umlaufsinn haben.

Da die äußere Abbremsung gemäß dem zuvor gegebenen Bilde einen axialsymmetrischen Cha-

25 L. B i e rm a n n, Ergebn. exakt. Naturwiss. 21, 1 [1945] S. 21. rakter hat, die molekulare Viskosität (einschließlich der Strahlungsviskosität) als eine Funktion der Temperatur und der Dichte dagegen eine im wesentlichen radialsymmetrische Verteilung besitzt, wird die von beiden abhängende Dicke der rotationsinstabilen Zone nach den Polen zu abnehmen. Die Rotationsinstabilität beeinflußt die Temperatur- und Dichteschichtung sowie den Energietransport, und da dieser Einfluß in der Äquatorebene demnach stärker ist bzw. tiefer reicht als in höheren heliographischen Breiten, erscheint es verständlich, daß innerhalb des instabilen Bereiches noch eine großräumige Zirkulation besteht, die diesen Unterschied kompensiert, obwohl die hiermit zusammenhängenden Fragen noch nicht genügend geklärt sind. Der Beobachtung nach müßte diese Zirkulation, wie es auch dem Bjerknesschen Bilde entspricht, in der Äquatorebene einwärts gerichtet sein, in der inneren Zone polwärts und an der Oberfläche äquatorwärts fließen und so das dort beobachtete Rotationsgesetz bewirken. Außerdem werden durch diese Zirkulation die einzelnen Wirbelringe mitgeführt. Zu der Zeit und an der Stelle, an der sie dabei der Oberfläche am nächsten kommen (nach der Beobachtung bei etwa $15^{\circ}$ heliographischer Breite), wird dann die Wahrscheinlichkeit für ein Durchbrechen der Wirbel bis zur Oberfläche und damit die Häufigkeit der Sonnenflecken am größten sєin.

Die Periode der Sonnenfleckenhäufigkeit muß nach dieser Vorstellung von der Strömungsgeschwindigkeit sowie von den Durchmessern und von der Zahl der Sonnenfleckenwirbel in der rotationsinstabilen Zone abhängen. Nimmt man mit Bjerknes nur zwei Wirbelringe in jeder Hemisphäre an, so bedeutet dies, daß die Geschwindigkeit der in der rotationsinstabilen Zone aufsteigenden Strömung ebenso groß sein muß wie die beobachtete Geschwindigkeit der absteigenden Strömung in der WKZ. Für den Fall aber, daß das Volumen der inneren Zone ebenso groß ist wie das der äußeren Zone oder größer, muß wegen der nach innen zunehmenden Dichte die Strömungsgeschwindigkeit in der inneren Zone um einige Größenordnungen kleiner sein, und man wird zu der Annahme gezwungen, daß in der rotationsinstabilen Zone mehrere Wirbelringe übereinander liegen, deren Zahl und Durchmesser mit der Geschwindigkeit der aufsteigenden Strömung und der beobachteten mittleren Sonnenfleckenperiode übereinstimmen müssen. 
Eine exakte quantitative Begründung dieser Vorstellung ist bei den $\mathrm{z}$. Zt. noch vorhandenen Unsicherheiten in der Theorie des inneren Aufbaues der Sonne nicht möglich, außerdem müßten dabei notwendig auch die elektromagnetischen Kräfte mit in Betracht gezogen werden, was eine weitere Ausbildung der Theorie der inneren Magnetfelder der Sonne voraussetzt. So soll abschließend nur noch kurz gezeigt werden, daß die in den Sonnenflecken beobachteten Magnetfelder ausreichen, um die Existenz stationärer Strömungsformen, wie sie die postulierten Wirbelringe darstellen, auch in an sich turbulenten Gebieten zu begründen.

Wie Cow ling ${ }^{26}$ gezeigt hat, ist die Zeit, in der Magnetfelder der in den Sonnenflecken beobachteten Stärke auf- bzw. abgebaut werden können, von der Größenordnung einiger hundert Jahre. Es bleibt daher nur zu zeigen übrig, daß durch diese Magnetfelder eine ausreichende Persistenz der mit ihnen behafteten Sonnenfleckenwirbel gegenüber der Turbulenz in der rotationsinstabilen Zone und in der WKZ gewährleistet ist. Fordert man daher, daß die magnetische Energiedichte größer sei als die turbulente Energiedichte,

$$
\frac{H^{2}}{8 \pi}>\frac{1}{2} \varrho w^{2}
$$

und nimmt für die Turbulenzgeschwindigkeit in dér rotationsinstabilen Zone entsprechend (3.13) $w \approx 2 \omega_{0} h$ und in der WKZ $w \approx 1 \mathrm{~km} / \mathrm{sec}$ an, so erhält man folgende Bedingungen für die

\begin{tabular}{|c|c|c|}
\hline & S-Lösung & K-Lösung \\
\hline WKZ & $\begin{aligned} d_{1} & =400 \mathrm{~km} \\
\bar{\varrho} & =10^{-6,4} \mathrm{~g} / \mathrm{cm}^{3} \\
H & >200 \mathrm{Gau} \beta\end{aligned}$ & $\begin{aligned} d_{1} & =10000 \mathrm{~km} \\
\bar{\varrho} & =10-3,0 \mathrm{~g} / \mathrm{cm}^{3} \\
H & >10000 \mathrm{Gau} \beta\end{aligned}$ \\
\hline $\begin{array}{c}\text { rotations- } \\
\text { instabile } \\
\text { Zone }\end{array}$ & $\begin{aligned} d_{2} & =23000 \mathrm{~km} \\
\bar{\varrho} & =10^{-5,5} \mathrm{~g} / \mathrm{cm}^{3} \\
H & >5 \mathrm{Gau} \Omega\end{aligned}$ & 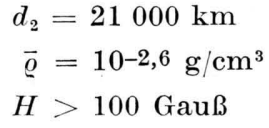 \\
\hline
\end{tabular}

Ein Vergleich der sich für die WKZ ergebenden Mindestwerte der magnetischen Feldstärke mit den beobachteten Werten $(300-3000$ Gauß) zeigt, daß mit ziemlicher Sicherheit die der K-Lösung

26 T. G. Cowling, Monthly Notices Roy. astronom. Soc. 106, 218 [1946]. entsprechende materielle Dichte in den äußeren Teilen der Sonne um etwa zwei Zehnerpotenzen $\mathrm{zu}$ hoch sein dürfte, und daß vielleicht allgemein die Turbulenzgeschwindigkeit mit $1 \mathrm{~km} / \mathrm{sec}$ etwas zu hoch angenommen wurde. Da außerdem in der rotationsinstabilen Zone schon verhältnismäßig geringe Feldstärken genügen, um die Erhaltung stationärer mit Magnetfeldern behafteter Zirkulationen auch in einer an sich turbulenten Zone zu gewährleisten, erscheinen die Schwierigkeiten, die sich am Ende des vorigen Abschnittes ergaben, wesentlich gemildert.

\section{Das Rotationsgesetz im tieferen Innern}

\section{Der Gesamtdrehimpuls der Sonne}

Nach allem, was man somit weiß, ist im tieferen Innern der Sonne - etwa innerhalb $r=0,80 R-$ sowohl die Viskosität wie die Geschwindigkeit der meridionalen Zirkulation als auch der Einfluß des Strahlungsstromes und der einer äußeren $\mathrm{Ab}$ bremsung zu klein, um innerhalb des Entwicklungsalters der Sonne eine definierte Verteilung der Winkelgeschwindigkeit bewirkt zu haben. Diese wird sich daher im einzelnen nicht angeben lassen, und man wird versuchen müssen, das Rotationsgesetz des Sonneninneren wenigstens einzugrenzen.

Die äußeren Teile der Sonne sind im Laufe der Zeit mit Sicherheit abgebremst worden, wenn nicht durch das interplanetarische Medium, dann wenigstens durch die Bremswirkung des Strahlungsstromes. Da sich kosmogonisch keine Gründe dafür angeben lassen, daß die Winkelgeschwindigkeit weiter nach innen hin abnimmt, wäre eine untere Grenze für das Rotationsgesetz dadurch gegeben, daß man annimmt, das Sonneninnere rotiere annähernd starr mit der Winkelgeschwindigkeit, die sich für die innere Grenze des der Abbremsung unterliegenden Bereiches ergibt.

Eine obere Grenze für das Rotationsgesetz, d. h. für die Zunahme der Winkelgeschwindigkeit im tieferen Innern ist dadurch gegeben, daß eine dementsprechend stärkere Abplattung der inneren Teile der Sonne sich in der Gestalt der Oberfläche als einer Äquipotentialfläche und in der Planetenbewegung bemerkbar machen müßte. Nun zeigt die Sonnenoberfläche innerhalb einer Meßgenauig- 
keit von 0 "1 bis $0^{\prime \prime} 01$ keine beobachtbare Abplattung ${ }^{27,28}$. Das bedeutet

$$
\frac{\Delta R}{R}=\left[\frac{a-c}{a}\right]_{0}<10^{-4}
$$

Andererseits ergibt sich aus der beobachteten Perihelverschiebung der Merkurbahn nach Abzug des relativistischen Effektes für das Verhältnis der Hauptträgheitsmomente der Sonne ein Wert, der ebenfalls praktisch durch die Grenze der Meßgenauigkeit bestimmt ist, nämlich ${ }^{29}$ )

$$
\frac{A-C}{A}<0,5 \cdot 10^{-4} \text {. }
$$

Für ein homogenes Rotationsellipsoid gilt bei hinreichend kleinem Unterschied in den Achsen

$$
\frac{A-C}{A}=\frac{1}{2} \frac{a^{2}-c^{2}}{a^{2}} \approx \frac{a-c}{a} .
$$

Die beiden Kriterien entsprechen demnach einem gleichen Grad von Meßgenauigkeit.

Um ein allgemeines Bild von der dadurch gegebenen Beschränkung der Winkelgeschwindigkeit im Innern der Sonne zu erhalten, seien folgende vereinfachende Annahmen gestattet:

Betrachtet man erstens den Unterschied in den Hauptträgheitsmomenten als nur von einem schneller rotierenden und daher stärker abgeplatteten Kern mit der Masse $M_{\mathrm{K}}$, dem Radius $r_{\mathrm{K}}$, und der Exentrizität

$$
e_{\mathrm{K}}^{2}=\left[\frac{a^{2}-c^{2}}{a^{2}}\right]_{\mathrm{K}}
$$

herrührend, so ist die letztere nach (5.2) und (5.3)

$$
e_{\mathrm{K}}^{2}<10^{-4} \frac{A}{A_{\mathrm{K}}} \text {. }
$$

Nimmt man weiter an, daß sowohl der Kern wie die ihn umgebende Hülle von zwar verschiedener, aber in sich homogener Dichte seien, so gibt

und

$$
A_{\mathrm{K}}=\frac{2}{5} M_{\mathrm{K}} a_{\mathrm{K}}^{2}
$$

$$
A=A_{\mathrm{K}}+\frac{2}{5}\left(\frac{M-\dot{M}_{\mathrm{K}}}{R^{3}-r_{\mathrm{K}}^{3}}\right)\left(R^{3} a^{2}-r_{\mathrm{K}}^{3} a_{\mathrm{K}}^{2}\right)
$$

in (5.4) eingesetzt mit $a \approx R$ und $a_{\mathrm{K}} \approx r_{\mathrm{K}}$

$e_{\mathrm{K}}^{2}<10^{-4}\left\{1+\left(\frac{M}{M_{\mathrm{K}}}-1\right) \frac{\left(R / r_{\mathrm{K}}\right)^{2}-\left(r_{\mathrm{K}} / R\right)^{3}}{1-\left(r_{\mathrm{K}} / R\right)^{3}}\right\}$.

27 M. Waldmeier, Ergebnisse und Probleme der Sonnenforschung, Akadem. Verlagsgesellschaft Leipzig 1941, S. $55 \mathrm{ff}$.

28 B. M eyermann, Astronom. Nachr. 269, 114 [1939]; 279, 45 [1950].
Der konvektive Kern der Sonne hat nach Kellers Rechnung eine Masse von 0,08 $M$ und einen Radius von $0,075 R$, damit gilt für ihn

$$
e_{\mathrm{K}}^{2}<0,20 \text {. }
$$

Nimmt man drittens an, daß dieser Kern starr rotiere, so ist die Exzentrizität mit der Winkelgeschwindigkeit durch die für die Mac Laurin schen Sphäroide geltende Beziehung

$\frac{\omega^{2}}{2 \pi \mathrm{G} \varrho}=\frac{3-2 e^{2}}{e^{3}} \sqrt{1-e^{2}} \arcsin e-3\left(\frac{1}{e^{2}}-1\right)$

verknüpft, und man erhält

$$
\left[\frac{\omega^{2}}{2 \pi \mathrm{G} \varrho}\right]_{\mathrm{K}}<0,055
$$

bzw.

$$
\omega_{\mathrm{K}} / \omega_{0}<630, \text { d. h. } \frac{\overline{\partial \ln \omega}}{\partial \ln r}>-2,5 .
$$

Von den gemachten Annahmen ist die erste die gewaltsamste. Bei einer natürlicherweise anzunehmenden kontinuierlichen Zunahme der Winkelgeschwindigkeit würde aber deren Einschränkung noch erheblich stärker.sein müssen. Man wird daher nicht fehl gehen, wenn man als obere Grenze für die Zunahme der Winkelgeschwindigkeit nach innen

$$
\overline{\frac{\partial \ln \omega}{\partial \ln r}}>-2,0
$$

annimmt.

Tatsächlich sind auch der Zustand annähernd starrer Rotation und der Zustand eines ausgeglichenen Rotationsmomentes die beiden Grenzfälle, die sich kosmogonisch begründen lassen, wenn in einem früheren Entwicklungsstadium der Sonne entweder die Viskosität oder die meridionalen Strömungen wesentlich stärker waren als heute und damit die im Innern noch vorhandene „Urverteilung“ der. Winkelgeschwindigkeit bestimmt haben.

An der Oberfläche ist

$$
\left[\frac{\omega^{2}}{2 \pi \mathrm{G} \bar{\varrho}}\right]_{0}=10^{-4,84} \text {. }
$$

Und da die innerhalb $r=0,80 R$ gelegenen Teile noch praktisch die ganze Masse umfassen, muß für sie nach (5.5) gelten

29 Nach freundlicher Mitteilung von Herrn Prof. Siedentopf, Tübingen, vgl. auch Clemens ${ }^{29 a}$. 29a G. M. Clem en s, Rev. mod. Physic. 19, 361 [1947]. 


$$
\frac{\omega^{2}}{2 \pi \mathrm{G} \varrho}<\frac{2}{3} \cdot 10^{-4} \text {, d. h. } \omega / \omega_{0}<3,0 .
$$

Dies gilt für ein homogenes Rotationsellipsoid. Bei Berücksichtigung der Dichtekonzentration der Sonne erhöht sich der Maximalwert für $\omega$ auf etwa das 10-fache ${ }^{29}$ a, so daß die sich für die SLösung ergebende Zunahme der Winkelgeschwindigkeit auf $\omega \approx 20 \omega_{0}$ (Abb. 7) ebenfalls einen vernünftigen Grenzwert darstellt, der kaum wesentlich überschritten werden dürfte.

Damit besteht nun die Möglichkeit, auch die Grenzen anzugeben, in denen etwa der Gesamtdrehimpuls der Sonne liegen wird. Unter Annahme eines Potenzgesetzes für $\omega$ und $\varrho$

$$
\omega=\omega_{0}\left(\frac{r}{R}\right)^{k}, \quad \varrho=\varrho_{c}\left(\frac{R-r}{R}\right)^{m}
$$

erhält man für den Gesamtdrehimpuls

$$
\begin{aligned}
D & =\int \omega r^{2} \sin ^{2} \vartheta \mathrm{d} M \\
& =\frac{8 \pi}{3} R^{5} \omega_{0} \varrho_{c} \int_{0}^{1}(1-x)^{m} x^{4+k} \mathrm{~d} x
\end{aligned}
$$

mit $x=r / R$, und nach der Integration die Summe

$$
\begin{gathered}
D=2 \omega_{0} M R^{2}\left(\frac{\varrho_{c}}{\varrho_{m}}\right) \\
\cdot\left\{\frac{1}{5+k}+\sum_{v=1}^{m}(-1)^{v} \frac{m(m-1) \ldots(m-v+1)}{v !(5+k+v)}\right\} .
\end{gathered}
$$

Für das O-Modell ist $\varrho_{c} / \varrho_{m}=229,9$ und die

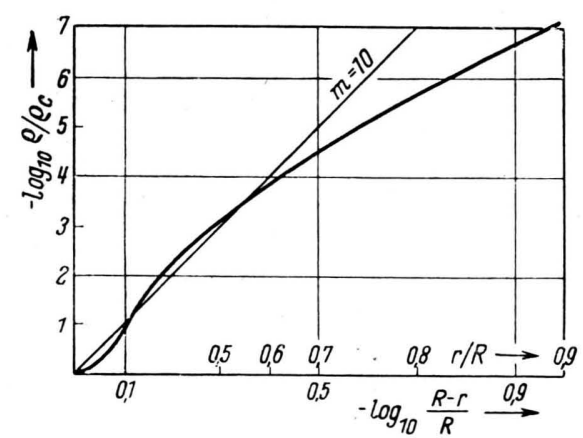

Abb. 9. Die Dichteverteilung für das O-Modell.
Dichteverteilung läßt sich innerhalb eines Radius $r=0,6 R$, der $99,6 \%$ der Masse umfaßt, mit guter Näherung durch $m=10$ darstellen (s. Abb. 9 ). Indem man als Grenzen für das Rotationsgesetz gemä $\beta$ dem oben Gesagten $k=0$ und $k=-2$ sowie $k=0, \omega \approx 20 \omega_{0}$ in (5.15) einsetzt, erhält man folgende Werte für den Gesamtdrehimpuls in $\mathrm{cm}^{2} \mathrm{~g} \mathrm{sec}^{-1}$

\begin{tabular}{c|cc}
\hline$\omega / \omega_{0}$ & $k=0$ & $k=-2$ \\
\hline 1 & $10^{47,9}$ & $10^{49,2}$ \\
20 & $10^{49,2}$ & -
\end{tabular}

Für die beiden Grenzfälle ergibt sich der gleiche Wert, den man außerdem auch bei Annahme homogener Dichte und starrer Rotation mit der an der Oberfläche beobachteten Winkelgeschwindigkeit erhalten würde, $D=2 / 5 \omega_{0} M R^{2}=10^{49,05}$ $\mathrm{cm}^{2} \mathrm{~g} \mathrm{sec}^{-1}$. Die Dichtekonzentration der Sonne macht auch in diesem Falle etwa eine Zehnerpotenz aus. Diese numerische Übereinstimmung muß als ein Zufall angesehen werden schon wegen der Unsicherheit der Unterlagen und des ganzen Rechenverfahrens. Man kann sich aber überlegen, daß eine genaue Rechnung für die beiden kosmogonisch möglichen Grenzfälle einer annähernd starren Rotation und eines weitgehend ausgeglichenen Rotationsmomentes denselben Wert ergeben sollte, weil es dem Stern bei zu vernachlässigenden äußeren Kräften möglich sein müßte, seinen Rotationszustand innerhalb dieser beiden Grenzen zu variieren, ohne daß sich sein Gesamtdrehimpuls dabei ändert.

Der Verfasser ist der Notgemeinschaft der Deutschen W issenschaft für die Gewährung eines Forschungsstipendiums zu großem Dank verpflichtet, ebenso Herrn Prof. L. Biermann für die Anregung und ständige Förderung dieser Untersuchung. Herrn Prof. M.S chwarzschild danke ich für wertvolle Hinweise, den Herren Lüst, Schlüter und Tillmann für viele Diskussionen. 